\title{
THE FORWARD AND BACKWARD SHIFT ON THE LIPSCHITZ SPACE OF A TREE
}

\author{
RUBÉN A. MARTÍNEZ-AVENDAÑO AND EMMANUEL RIVERA-GUASCO
}

\begin{abstract}
We initiate the study of the forward and backward shifts on the Lipschitz space of an undirected tree, $\mathcal{L}$, and on the little Lipschitz space of an undirected tree, $\mathcal{L}_{0}$. We determine that the forward shift is bounded both on $\mathcal{L}$ and on $\mathcal{L}_{0}$ and, when the tree is leafless, it is an isometry; we also calculate its spectrum. For the backward shift, we determine when it is bounded on $\mathcal{L}$ and on $\mathcal{L}_{0}$, we find the norm when the tree is homogeneous, we calculate the spectrum for the case when the tree is homogeneous, and we determine, for a general tree, when it is hypercyclic.
\end{abstract}

\section{INTRODUCTION}

In 11, Colonna and Easley introduced the Lipschitz space of a tree, $\mathcal{L}$. This is the Banach space of complex-valued functions on a rooted, countably infinite, locally finite and undirected tree (from now on simply referred to as a tree) which are Lipschitz functions, when the tree is endowed with the edge-counting metric. This space may be considered as the discrete analogue of the classical Bloch space: the space of functions $f: \mathbb{D} \rightarrow \mathbb{C}$ which are Lipschitz when the unit disk $\mathbb{D}$ is given the hyperbolic or Bergman metric (see, e.g., 24]) and the set of complex numbers $\mathbb{C}$ is given the usual Euclidean metric.

As it turns out, the Lipschitz space of the tree is, roughly speaking, the space of funtions on the tree whose "derivative" remains bounded on the tree. Therefore, there is also the little Lipschitz space, $\mathcal{L}_{0}$, defined as the space of functions on the tree whose "derivative" tends to zero when far away from the root of the tree (i.e., on the "boundary" of the tree).

The motivation for investigating spaces of functions on trees comes mainly from harmonic analysis. Early studies of harmonic functions on regular trees were done by Cartier in [7, 8. Also, Cohen and Colonna studied the Bloch space of harmonic functions on a regular tree in [9], characterizing several properties of functions on this space. Later, in [10] Cohen and Colonna showed how to embed certain homogeneous trees in the hyperbolic disk in a "nice way": for example, in such a way that bounded harmonic functions on the disk correspond to harmonic functions on the tree.

Several operators on the Lipschitz space of a tree have been studied. For instance, in [11, Colonna and Easley characterize boundedness of multiplication operators on $\mathcal{L}$ and on $\mathcal{L}_{0}$, as well as establishing other operator-theoretical properties of such operators. In [4, Allen, Colonna and Easley study properties of the composition operators on the Lipschitz space of a tree. There have also been several studies of multiplication and other operators defined on $\mathcal{L}$ and on other Banach spaces on trees [1, 2, 3, 12, 13, 14.

The shift operators (both the forward and backward shifts) on $\ell^{p}$ have been studied for a long time. There are several reasons why researchers have been interested in shift operators: one of them is that they provide a wealth of examples and counterexamples in operator theory (see, e.g., [23]). In [19], Jabłoński, Jung and Stochel initiated the study of shifts on directed trees. In their paper, they investigate several operator theoretic properties of weighted (forward) shifts on the $L^{2}$ space of an infinite directed tree. Later, in [21, the first author defined (inspired by a result in 19]) the backward shift operator on a weighted $L^{p}$ space of a directed tree and characterized its hypercyclicity.

The study of hypercyclic operators goes as far back as the papers of Birkhoff [6] and MacLane [20], but the first example of a hypercyclic operator on a Banach space was given by Rolewicz 22]: it is a multiple of the backward shift on $\ell^{p}$. For the basic definitions and the history of hypercycicity, we recommend the texts [17] and [5].

Date: January 29, 2020.

2010 Mathematics Subject Classification. 47A16, 47B37, 05C05, 05C63.

Key words and phrases. Hypercyclicity, Lipschitz space, trees, shifts.

We would like to thank the reviewers for their suggestions, which greatly improved this paper. The first author's research is partially supported by the Asociación Mexicana de Cultura A.C. 
The purpose of this paper is to introduce the study of the forward and backward shift operator on the Lipschitz space $\mathcal{L}$ and on the little Lipschitz space $\mathcal{L}_{0}$. The paper is organized as follows. After giving the basic definitions and notations we will use throughout this paper in Section 2, we define the forward and backward shifts in Section 3. We observe that the forward shift is always an isometry, when the tree is leafless, and find its spectrum when it acts on $\mathcal{L}$ and on $\mathcal{L}_{0}$. Also, we establish that the backward shift is the adjoint operator of the forward shift. In Section 4 , we give a sufficient and necessary condition to ensure that the backward shift is bounded: it turns out the backward shift is bounded exactly when the tree is homogeneous by sectors. We summarize this characterization in Theorem 4.10, and we also find some lower estimates for the norm. Later, in Section [5 we find the value of the norm of the backward shift and the value of the norm for powers of the backward shift, in the case of homogeneous trees. In Section 6, we obtain the spectrum for the backward shift in the case where the tree is homogeneous, both for $\mathcal{L}$ (Theorem 6.2) and for $\mathcal{L}_{0}$ (Theorems 6.3 and 6.4). Lastly, in Section 7, we establish that the forward shift can never be hypercyclic, but the backward shift is hypercyclic exactly when the tree has no free ends (Theorem 7.7): this result is analogous to the one found in [21].

\section{Preliminaries}

As is customary, $\mathbb{N}, \mathbb{N}_{0}, \mathbb{R}, \mathbb{C}$ and $\mathbb{D}$ will denote the set of natural numbers, the set of nonnegative integers, the set of real numbers, the set of complex numbers, and the open unit disk in $\mathbb{C}$ centered at the origin, respectively.

Recall that a graph $G=(V, E)$ consists of a nonempty set of vertices $V$ and a set of edges $E \subseteq$ $\{\{u, v\}: u, v \in V, u \neq v\}$. In this paper, the set of vertices $V$ will always be countably infinite. If $\{u, v\} \in E$, we say that $u$ and $v$ are adjacent and we denote this by $u \sim v$. For each $u \in V$, the degree of $u$, denoted by $\operatorname{deg}(u)$, is the number of vertices adjacent to $u$. In this paper, all of our graphs will be locally finite; i.e., $\operatorname{deg}(u)<\infty$ for every $u \in V$.

A path of lenght $n$ joining two vertices $u$ and $v$ is a finite sequence of $n+1$ distinct vertices $u=u_{0} \sim$ $u_{1} \sim u_{2} \sim \cdots \sim u_{n}=v$. A graph is a tree if for each pair of vertices there is one and only one path between them. In this paper, for a tree $T$ we will denote its set of vertices also by the letter $T$, which should cause no confusion.

Every tree $T$ we consider here has a distinguished vertex, which we call the root of $T$ and denote by o. For a tree $T$, we denote by $\mathrm{d}(u, v)$ the length of the unique path between the vertices $u, v \in T$. For $v \in T$ we use the notation $|v|:=\mathrm{d}(\mathrm{o}, v)$. We also denote by $T^{*}$ the set of all vertices minus the root; i.e., $T^{*}:=\{v \in T:|v| \geq 1\}$. Ocasionally, we will denote by $T^{* *}$ the set $\{v \in T:|v| \geq 2\}$.

For each $v \in T^{*}$, we define the parent of $v$, denoted by $\operatorname{par}(v)$, as the unique vertex $w$ in the path from o to $v$ with $|w|=|v|-1$. Observe that every vertex in $T$ has a parent, except for the root o. Inductively, for

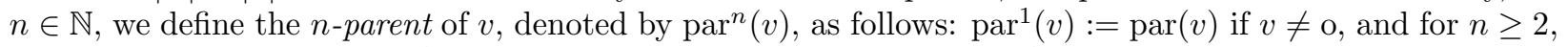
we set $\operatorname{par}^{n}(v):=\operatorname{par}\left(\operatorname{par}^{n-1}(v)\right)$, if $v$ has a $(n-1)$-parent and $\operatorname{par}^{n-1}(v) \neq 0$. The set of all vertices that have $n$-parents is denoted by $T^{n}$.

Note: We should point out that with the above definitions we are giving the tree a directed structure, in the manner of the definitions in [19: each edge can be thought of as a directed edge going to a vertex $v \in T^{*}$ from its parent $\operatorname{par}(v)$. Also, we have chosen a fixed vertex and we called it a root, which has no parent and thus coincides with the definition of a root in [19] (and hence it is unique). We choose not to follow this point of view in this work, since the spaces we study ahead, were originally defined on undirected trees.

If $w$ is the parent of $v$, we say that $v$ is a child of $w$ and we denote the set of all children of $w$ by $\operatorname{Chi}(w)$. If $w$ is the $n$-parent of $v$ we say that $v$ is an $n$-child of $w$ and we denote the set of all $n$-children of $w$ by $\mathrm{Chi}^{n}(w)$. For a vertex $v$, we denote by $\gamma(v)$ the number of children it has; i.e., $\gamma(v)$ is the cardinality of $\operatorname{Chi}(v)$. Also, $\gamma(v, n)$ is the number of $n$-children of $v$; i.e., $\gamma(v, n)$ is the cardinality of $\mathrm{Chi}^{n}(v)$. We will say a tree is homogeneous of order $\gamma$ if $\gamma(v)=\gamma$ for all $v \in T$ (this differs a bit from the use of the term in the literature).

If a vertex $v \in T$ satisfies that $\gamma(v)=0$ (i.e., $v$ has no children) we will say that $v$ is a leaf of $T$. A tree with no leaves will be called leafless. Observe that in a leafless tree, every vertex is the parent of some other vertex.

Lastly, for every $v \in T$, we denote by $S_{v}$ the sector determined by $v$, which consists of $v$ and all its $n$ children; i.e., $S_{v}:=\bigcup_{n=0}^{\infty} \operatorname{Chi}^{n}(v)$, where we will agree that $\operatorname{Chi}^{0}(v)=\{v\}$ and $\operatorname{Chi}^{1}(v)=\operatorname{Chi}(v)$. Sometimes we will refer to a sector as a subtree. 


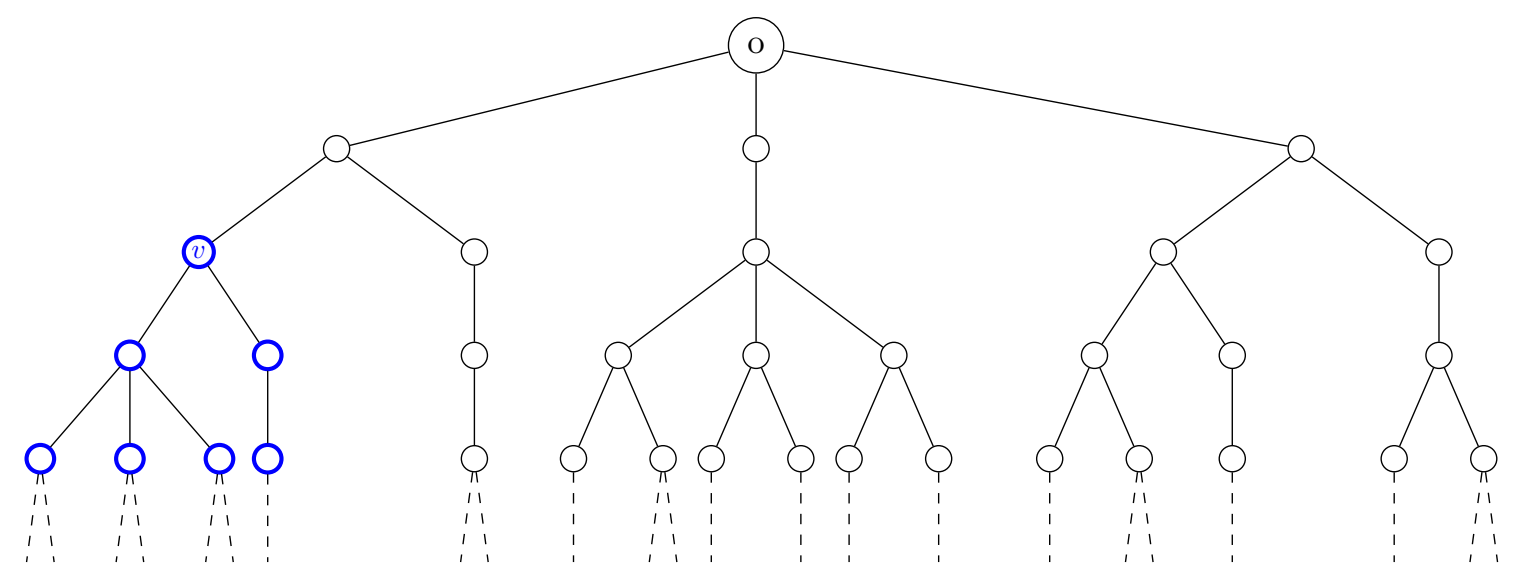

Figure 1. Sector at $v$ : vertices in blue (and below).

Let $T$ be a countably infinite, locally finite tree. We denote by $\mathcal{F}$ the set of all functions $f: T \rightarrow \mathbb{C}$. In [1], Colonna and Easley define the Lipschitz space of a tree as follows.

Definition 2.1. The Lipschitz space of $T$ is the set of all complex-valued Lipschitz functions on $T$; i.e., $f \in \mathcal{F}$ is Lipschitz if

$$
\sup _{u \neq v} \frac{|f(u)-f(v)|}{\mathrm{d}(u, v)}<\infty .
$$

In [11, Colonna and Easley show that

$$
\sup _{u \neq v} \frac{|f(u)-f(v)|}{\mathrm{d}(u, v)}=\sup _{v \in T^{*}}|f(v)-f(\operatorname{par}(v))|
$$

and hence the Lipschitz space consists of all functions for which

$$
\sup _{v \in T^{*}}|f(v)-f(\operatorname{par}(v))|<\infty .
$$

For $f \in \mathcal{F}$, we define $f^{\prime}$ as the function

$$
f^{\prime}(v)= \begin{cases}f(\mathrm{o}), & \text { if } v=\mathrm{o} \\ f(v)-f(\operatorname{par}(v)), & \text { if } v \neq 0 .\end{cases}
$$

Thus $f$ is in the Lipschitz space if $f^{\prime}$ is bounded. We denote by $\mathcal{L}$ the set of all such functions endowed with the norm

$$
\|f\|=\sup _{v \in T}\left|f^{\prime}(v)\right| .
$$

Colonna and Easley showed in [1] that $\mathcal{L}$, with an equivalent norm, is a Banach space. We use the present norm, following [13, to make some calculations cleaner.

The following lemma, which we will use later, can be easily obtained using the results in 11. For the sake of completeness, we give the proof here (which is a slight modification of the proof in [11) since we are using a different norm.

Lemma 2.2. Let $f \in \mathcal{L}$ and $v \in T$. Then

$$
|f(v)| \leq(|v|+1)\|f\| .
$$

Proof. First, we claim that if $f \in \mathcal{L}, f(\mathrm{o})=0$ and $\|f\| \leq 1$, then $|f(v)| \leq|v|$ for every $v \in T$. We prove this by induction on $|v|$. It is clear that the claim is true for $|v|=0$. Assume that the claim holds for all $v \in T$ with $|v|=n \in \mathbb{N}_{0}$, and let $w \in T$ with $|w|=n+1$. Then

$$
|f(w)| \leq|f(w)-f(\operatorname{par}(w))|+|f(\operatorname{par}(w))|=\left|f^{\prime}(w)\right|+|f(\operatorname{par}(w))| \leq\|f\|+|\operatorname{par}(w)| \leq 1+n=|w|,
$$

which completes the induction step and finishes the proof of the claim. 
Now, observe that the lemma is trivial if $f$ is the zero function. So assume that $f$ is not identically zero. For the moment, assume $\|f\|=1$. Define $g$ as $g(v)=f(v)-f(\mathrm{o})$. Clearly, $g \in \mathcal{L}$. Observe that $g^{\prime}(v)=f^{\prime}(v)$ for all $v \in T^{*}$ and $g(\mathrm{o})=g^{\prime}(\mathrm{o})=0$. Hence

$$
\|g\|=\sup _{v \in T}\left|g^{\prime}(v)\right|=\sup _{v \in T^{*}}\left|f^{\prime}(v)\right| \leq\|f\|=1,
$$

so aplying the claim to $g$ we obtain

$$
|f(v)-f(\mathrm{o})|=|g(v)| \leq|v|
$$

for every $v \in T$. But from this we obtain

$$
|f(v)| \leq|f(v)-f(\mathrm{o})|+|f(\mathrm{o})| \leq|v|+1,
$$

which proves the theorem for functions $f \in \mathcal{L}$ with $\|f\|=1$. Now let $f$ be an arbitrary nonzero function in $\mathcal{L}$. Applying the previous argument to $\frac{f}{\|f\|}$ we obtain

$$
\frac{|f(v)|}{\|f\|} \leq|v|+1
$$

which finishes the proof.

Also of interest is the little Lipshitz space of $T$, denoted by $\mathcal{L}_{0}$, defined as the set of all $f \in \mathcal{F}$ for which

$$
\lim _{|v| \rightarrow \infty} f^{\prime}(v)=0
$$

Clearly $\mathcal{L}_{0}$ is a subset of $\mathcal{L}$ and it can be shown (see [11) that it is a separable closed subspace of $\mathcal{L}$.

In Section 7 we will talk about hypercyclicity. Recall that a bounded operator $A$ on a Banach space $\mathcal{B}$ is hypercyclic if there exists a vector $f \in \mathcal{B}$ (called a hypercyclic vector for $A$ ) such that the orbit of $f$ under $A$ is dense in the Banach space; i.e., the set

$$
\left\{f, A f, A^{2} f, A^{3} f, \ldots\right\}
$$

is dense in $\mathcal{B}$. Clearly, if $A$ is hypercyclic, then $\mathcal{B}$ must be separable. Also observe that if $f$ is a hypercyclic vector, then so is $A^{n} f$, for any $n \in \mathbb{N}$. Thus, if $A$ is hypercyclic, then the set of its hypercyclic vectors is dense in $\mathcal{B}$.

One way to prove that an operator is hypercyclic is to apply the hypercyclicity criterion. We include here the version we will use in this paper.

Theorem 2.3 (Hypercyclicity Criterion). Let $\mathcal{B}$ be a separable Banach space and $A$ a bounded operator on $\mathcal{B}$. Assume there exists a set $X$, dense in $\mathcal{B}$, and for each $n \in \mathbb{N}$ there exists a function $R_{n}: X \rightarrow \mathcal{B}$ such that, for every $f \in X$ we have

- $A^{n} f \rightarrow 0$ as $n \rightarrow \infty$,

- $R_{n} f \rightarrow 0$ as $n \rightarrow \infty$, and

- $A^{n} R_{n} f \rightarrow f$ as $n \rightarrow \infty$.

Then $A$ is hypercyclic.

The proof (of a more general version) of this theorem can be found in [17, p. 74]. A lot more information about the fascinating topic of hypercyclicity can be found in [17] and [5].

\section{THE FORWARD SHIFT AND ITS ADJOINT}

We now present the two main objects of study in this note. The first operator was originally defined, on $\ell^{2}(V)$, in [19].

Definition 3.1. Let $T$ be a a rooted, countably infinite and localy finite tree. The forward shift operator $S: \mathcal{F} \rightarrow \mathcal{F}$ is defined as

$$
(S f)(v)= \begin{cases}0, & \text { if } v=\mathrm{o} \\ f(\operatorname{par}(v)), & \text { if } v \neq \mathrm{o}\end{cases}
$$

It is clear that $S$ is a linear operator on $\mathcal{F}$. In [19, Proposition 3.4.1 (iii)], Jabłonski, Jung and Stochel found the form for the (Hilbert space) adjoint of $S$ on $\ell^{2}(V)$. Inspired by this result, the first author made the following definition in 21]. 
Definition 3.2. Let $T$ be a rooted, countably infinite and locally finite tree. The backward shift operator $B: \mathcal{F} \rightarrow \mathcal{F}$ is defined as

$$
(B f)(v)=\sum_{w \in \operatorname{Chi}(v)} f(w)
$$

where if $v$ has no children, the sum is understood to be empty and hence $(B f)(v)=0$.

Also, it is clear that $B$ is a linear operator on $\mathcal{F}$. We should point out that the operator $S+B$ is the adjacency operator (or adjacency matrix) of the graph $T$. We will see at the end of this section that $B$ is the Banach space adjoint of $S$ (on an appropriate space). We start with some results about the forward shift.

Theorem 3.3. Let $T$ be a rooted, countable infinite and locally finite tree and let $S$ be the forward shift. Then $S: \mathcal{L} \rightarrow \mathcal{L}$ is bounded and $\|S\| \leq 1$. If $T$ is leafless, then $\|S f\|=\|f\|$.

Proof. Let $f \in \mathcal{L}$. It is a straightforward calculation to check that $(S f)^{\prime}=S f^{\prime}$. Hence we get

$$
\|S f\|=\sup _{v \in T}\left|(S f)^{\prime}(v)\right|=\sup _{v \in T}\left|\left(S f^{\prime}\right)(v)\right|=\sup _{v \in T^{*}}\left|\left(S f^{\prime}\right)(v)\right|=\sup _{v \in T^{*}}\left|f^{\prime}(\operatorname{par}(v))\right| \leq \sup _{w \in T}\left|f^{\prime}(w)\right|=\|f\|,
$$

and hence $\|S\| \leq 1$. If $T$ is leafless, the the inequality in the expression above is an equality. Hence $S$ is an isometry, as desired.

The same result holds for $S$ as an operator on $\mathcal{L}_{0}$.

Theorem 3.4. Let $T$ be a rooted, countable infinite and locally finite tree and let $S$ be the forward shift. Then $S: \mathcal{L}_{0} \rightarrow \mathcal{L}_{0}$ is bounded and $\|S\| \leq 1$. If $T$ is leafless, then $\|S f\|=\|f\|$.

Proof. We first observe that if $f \in \mathcal{L}_{0}$, then $S f \in \mathcal{L}_{0}$. Indeed,

$$
\lim _{|v| \rightarrow \infty}(S f)^{\prime}(v)=\lim _{|v| \rightarrow \infty}\left(S f^{\prime}\right)(v)=\lim _{|v| \rightarrow \infty} f^{\prime}(\operatorname{par}(v))=\lim _{|w| \rightarrow \infty} f^{\prime}(w)=0 .
$$

Since $\mathcal{L}_{0}$ is a closed subspace of $\mathcal{L}$, it follows that $S$ is a bounded operator on $\mathcal{L}_{0}$. The calculation in the theorem above then shows that $\|S\| \leq 1$ and that $S$ is an isometry if $T$ is leafless.

If $T$ has leaves, we will show later (right after Proposition 3.5) that $S$ has nontrivial kernel (both as an operator on $\mathcal{L}$ and on $\mathcal{L}_{0}$ ) and hence it is not an isometry.

If $T$ is leafless, we have shown that $S$ is an isometry on $\mathcal{L}$. By a theorem of Koehler and Rosenthal (see, for example, [16, p. 11]) there exists a semi-inner product $[\cdot, \cdot]$ in $\mathcal{L}$ such that $[S f, S g]=[f, g]$ for all $f, g \in \mathcal{L}$. This fact raises a lot of questions about the structure of $\mathcal{L}$ with this seminorm. We plan to explore this in future research.

On the other hand, if $T$ has leaves, one may ask how "far" is $S$ from an isometry 1 It is not hard to see (for example, use Theorem 3.6 below and mimic the argument in [18, Problem 150]) that $\|S-U\|=2$, if $U$ is a surjective isometry. Is it possible that there exists a (non surjective) isometry $U$ such that $\|S-U\|<2$ ? We leave this question open for future research.

We should point out that if $T$ has leaves, we can define

$$
\mathcal{M}:=\{f \in \mathcal{L}: f(v)=0 \text { for all } v \in A\},
$$

where $A:=\left\{w \in T: w\right.$ is a leaf or $w=\operatorname{par}^{n}(v)$ for some leaf $v \in T$ and $\left.n \in \mathbb{N}\right\}$; i.e., $\mathcal{M}$ is the subspace of all functions that vanish on every leaf and every ancestor of a leaf. Then clearly $\mathcal{M}$ is an invariant subspace for $S$ (of finite codimension if $T$ has finitely many leaves) and $\left.S\right|_{\mathcal{M}}$ is an isometry. Thus, even if $T$ has leaves, there is a subspace in which $S$ acts isometrically.

We will now study the spectrum of $S$. We first show that the forward shift has no eigenvalues, not even on $\mathcal{F}$. Recall that for an operator $A$, the set of eigenvalues, the approximate point spectrum and the spectrum, are denoted by $\sigma_{\mathrm{p}}(A), \sigma_{\mathrm{ap}}(A)$, and $\sigma(A)$, respectively. (The relevant definitions can be found in, e.g., [15].)

Proposition 3.5. Let $T$ be a rooted, leafless, countably infinite and locally finite tree and let $S$ be the forward shift on $\mathcal{F}$. Then $\sigma_{\mathrm{p}}(S)=\varnothing$.

\footnotetext{
${ }^{1}$ We thank a referee for suggesting this question and for providing the reference in the previous paragraph.
} 
Proof. Let $f \in \mathcal{F}$. Clearly, $(S f)(v)=0$ for all $v \in T$ implies that $f(\operatorname{par}(v))=0$ for all $v \in T^{*}$ and hence $f(w)=0$ for all $w \in T$. Thus $\lambda=0$ is not eigenvalue.

Assume there exists $\lambda \neq 0$ such that $S f=\lambda f$, with $f \in \mathcal{F}$. Since $0=(S f)(\mathrm{o})=\lambda f(\mathrm{o})$ it follows that $f(\mathrm{o})=0$. Now, for every $v \in \operatorname{Chi}(\mathrm{o})$ we have $\lambda f(v)=(S f)(v)=f(\operatorname{par}(v))=f(\mathrm{o})=0$ and hence $f(v)=0$. Continuing in this manner, we obtain that $f(v)=0$ for all $v \in T$ and thus $\lambda$ is not an eigenvalue.

Observe that if the tree has a leaf, then 0 is an eigenvalue of $S$ as an operator on $\mathcal{F}$ : indeed, if $v$ is a leaf, then $S \chi_{\{v\}}=0$, where $\chi_{\{v\}}$ is the characteristic function of $v$. The proof above shows that, in this case, 0 is the unique eigenvalue of $S$.

As a corollary, it should be noted that in the leafless case, $S$ has no eigenvalues as an operator on $\mathcal{L}$ and as an operator on $\mathcal{L}_{0}$. If $T$ has a leaf, 0 is an eigenvalue for $S$ both on $\mathcal{L}$ and on $\mathcal{L}_{0}$, with eigenvector $\chi_{\{v\}}$. (By the way, this also shows that if $T$ has a leaf, then $S$ is not an isometry on $\mathcal{L}$ nor on $\mathcal{L}_{0}$; see Theorems 3.3 and 3.4).

In the leafless case, since $S$ is an isometry, its approximate point spectrum lies in the unit circle. Indeed, let $\lambda \in \mathbb{C}$ with $|\lambda| \neq 1$; then

$$
\|(S-\lambda) f\| \geq|\|S f\|-\|\lambda f\||=|1-| \lambda||\|f\|,
$$

and hence $S-\lambda$ is bounded below. Therefore, $\lambda \notin \sigma_{\text {ap }}(S)$.

The following theorem gives a full description of the spectrum of $S$ in the leafless case. It should be noted that it is known that the spectrum of a noninvertible isometry is always $\overline{\mathbb{D}}$ (e.g. [15, p. 213]), we prefer to give an independent proof since it gives more information about $S$.

Theorem 3.6. Let $T$ be a rooted, countable infinite and locally finite tree and let $S$ be the forward shift on $\mathcal{L}$ or on $\mathcal{L}_{0}$. Then, $\sigma(S)=\overline{\mathbb{D}}$. If $T$ is leafless, then $\sigma_{\text {ap }}(S)=\partial \mathbb{D}$.

Proof. Since $\|S\| \leq 1$, it follows that $\sigma(S) \subseteq \overline{\mathbb{D}}$. It is easily verified that the equation $S f=\chi_{\{0\}}$ has no solution $f \in \mathcal{F}$ (just evaluate at the root o), hence $0 \in \sigma(S)$.

Let $\lambda \in \mathbb{D}, \lambda \neq 0$. Then the equation

$$
(S-\lambda) f=\chi_{\{0\}}
$$

has a unique solution $f \in \mathcal{F}$ given by $f(v)=\frac{-1}{\lambda|v|+1}$, as a straightforward calculation shows. But in this case, $f^{\prime}(v)$, for $v \in T^{*}$, is given by

$$
f^{\prime}(v)=\left(1-\lambda^{-1}\right) \lambda^{-|v|} .
$$

But since $\lambda \in \mathbb{D}$, the function $f^{\prime}$ is unbounded, and thus $f \notin \mathcal{L}$ (and $f \notin \mathcal{L}_{0}$ ). Hence $S-\lambda$ is not surjective and thus $\mathbb{D} \backslash\{0\} \subseteq \sigma(S)$. It then follows that $\overline{\mathbb{D}} \subseteq \sigma(S)$ and hence $\sigma(S)=\overline{\mathbb{D}}$.

Lastly, assume $T$ is leafless. Recall that for any operator $A$ we have $\partial \sigma(A) \subseteq \sigma_{\text {ap }}(A)$ (e.g. [15, Prop. 6.7] and hence $\partial \mathbb{D}=\partial \sigma(S) \subseteq \sigma_{\text {ap }}(S) \subseteq \partial \mathbb{D}$ (since $S$ is an isometry, as noted above). Therefore $\sigma_{\text {ap }}(S)=\partial \mathbb{D}$, as desired.

If $T$ has leaves, as we showed before, 0 is an eigenvalue of $S$ and thus $\sigma_{\text {ap }}(S) \neq \partial \mathbb{D}$. Also observe that, as a corollary, we obtain that $\|S\|=1$, even in the case where $T$ has leaves.

In [13] it is shown that the dual space of $\mathcal{L}_{0}$ is (isometrically isomorphic to) the space $L^{1}(T)$ and the dual space of $L^{1}(T)$ is (isometrically isomorphic to) $\mathcal{L}$. Using the identification in [13] we can make the following observations.

Proposition 3.7. Let $T$ be a rooted, countable infinite and locally finite tree. If $S: \mathcal{L}_{0} \rightarrow \mathcal{L}_{0}$ is the forward shift, then $S^{*}: L^{1}(T) \rightarrow L^{1}(T)$ is given by $S^{*}=B$, where $B$ is the backward shift restricted to $L^{1}(T)$.

Proof. It is shown in [13] that, for every $f \in L^{1}(T)$, the functional $\Phi_{f}: \mathcal{L}_{0} \rightarrow \mathbb{C}$, defined as

$$
\Phi_{f}(g)=\sum_{v \in T} f(v) g^{\prime}(v),
$$


for each $g \in \mathcal{L}_{0}$, is bounded and the mapping $f \mapsto \Phi_{f}$ is an isometric isomorphism from $L^{1}(T)$ onto $\mathcal{L}_{0}^{*}$. Using this identification we obtain

$$
\begin{aligned}
\left(S^{*} \Phi_{f}\right)(g) & =\Phi_{f}(S g) \\
& =\sum_{w \in T} f(w)(S g)^{\prime}(w) \\
& =f(\mathrm{o})(S g)(\mathrm{o})+\sum_{w \in T^{*}} f(w)((S g)(w)-(S g)(\operatorname{par}(w))) \\
& =\sum_{v \in T^{*}} f(w)((S g)(w)-(S g)(\operatorname{par}(w)) \\
& =\sum_{w \in T^{*}} f(w) g(\operatorname{par}(w))-\sum_{w \in T^{* *}} f(w) g\left(\operatorname{par}^{2}(w)\right) .
\end{aligned}
$$

Observe that for every vertex $v \in T$ either $\operatorname{Chi}(v)$ is empty and hence

$$
\sum_{w \in \operatorname{Chi}(v)} f(w)=0
$$

or there are vertices $w \in T^{*}$ with $v=\operatorname{par}(w)$. Therefore,

$$
\sum_{w \in T^{*}} f(w) g(\operatorname{par}(w))=\sum_{v \in T} g(v)\left(\sum_{w \in \operatorname{Chi}(v)} f(w)\right) .
$$

Hence, Equation (1) implies that

$$
\begin{aligned}
\left(S^{*} \Phi_{f}\right)(g) & =\sum_{v \in T} g(v)\left(\sum_{w \in \operatorname{Chi}(v)} f(w)\right)-\sum_{v \in T^{*}} g(\operatorname{par}(v))\left(\sum_{w \in \operatorname{Chi}(v)} f(w)\right) \\
& =\left(\sum_{w \in \operatorname{Chi}(o)} f(w)\right) g(\mathrm{o})+\sum_{v \in T^{*}}\left(\sum_{w \in \operatorname{Chi}(v)} f(w)\right)(g(v)-g(\operatorname{par}(v))) \\
& =\sum_{v \in T}\left(\sum_{w \in \operatorname{Chi}(v)} f(w)\right) g^{\prime}(v) \\
& =\sum_{v \in T}(B f)(v) g^{\prime}(v) \\
& =\Phi_{B f}(g) .
\end{aligned}
$$

Hence $S^{*}$ can be identified with $B$ on $L^{1}(T)$.

Using the same technique as above, the following result can be shown.

Proposition 3.8. Let $T$ be a rooted, countable infinite and locally finite tree. If $B: L^{1}(T) \rightarrow L^{1}(T)$ is the backward shift, then $B^{*}: \mathcal{L} \rightarrow \mathcal{L}$ is given by $B^{*}=S$.

The above results show why we choose to call $B$ the "backward" shift, as an analogy of what happens with the classical forward and backward shifts on $\ell^{p}(\mathbb{N})$. We study this operator in greater depth in the next section.

\section{THE BACKWARD SHIFT}

It will turn out that the backward shift operator is not always bounded on $\mathcal{L}$ or on $\mathcal{L}_{0}$, as was the case for the forward shift. We will need the following definition (see Figure 2 for an example).

Definition 4.1. Let $T$ be a rooted, countably infinite and locally finite tree. We say that $T$ is homogenous by sectors (at the level $N$ ) if there exists $N \in \mathbb{N}_{0}$ such that for all $v \in T$ with $|v|=N$, we have $\gamma(v)=\gamma(w)$ for each $w \in S_{v}$. 


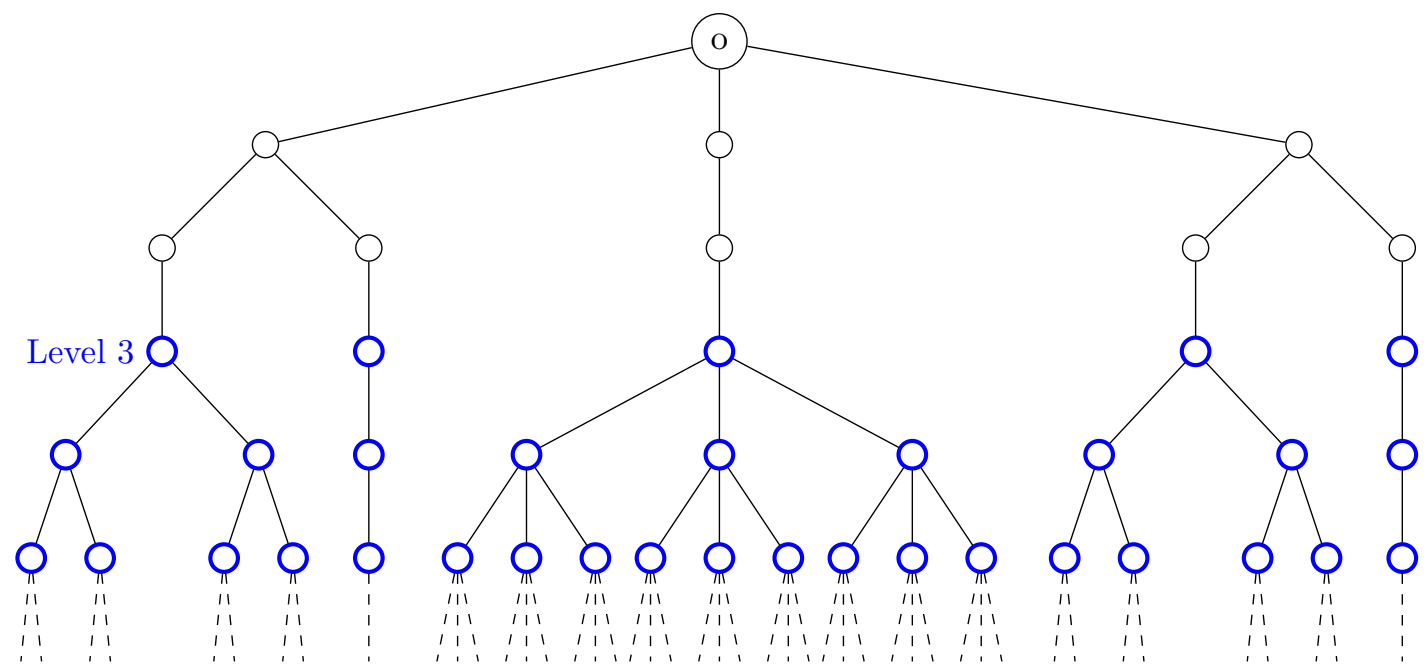

Figure 2. A tree which is homogeneous by sectors at the level 3.

Intuitively, a tree is homogeneous by sectors if after some level every subtree is a homogeneous tree. For a tree $T$, homogeneous by sectors at the level $N$, we define

$$
\Lambda:=\sup \left\{\gamma(v)+\gamma(\operatorname{par}(v))-1+|\gamma(v)-1|+|\gamma(v)-\gamma(\operatorname{par}(v))||v|: v \in T^{*}\right\} .
$$

Since the tree is homogeneous by sectors at the level $N$, it turns out the above supremum is finite and, in fact,

$$
\Lambda=\max \{\gamma(v)+\gamma(\operatorname{par}(v))-1+|\gamma(v)-1|+|\gamma(v)-\gamma(\operatorname{par}(v))||v|: 0<|v| \leq N\}
$$

As it turns out, $B$ is bounded if the tree is homogeneous by sectors and $\Lambda$ gives an estimate of the norm of $B$. In the corollary that follows the next proposition, we will find a more manageable estimate.

Proposition 4.2. Let $T$ be a rooted, countable infinite and locally finite tree. Assume that $T$ is homogeneous by sectors at the level $N$. Then $B$ is bounded on $\mathcal{L}$. Furthermore,

$$
\|B\| \leq \max \{2 \gamma(\mathrm{o}), \Lambda\} .
$$

Proof. First observe that

$$
(B f)^{\prime}(\mathrm{o})=(B f)(\mathrm{o})=\sum_{w \in \operatorname{Chi}(\mathrm{o})} f(w)=\sum_{w \in \operatorname{Chi}(\mathrm{o})}(f(w)-f(\operatorname{par}(w)))+\gamma(\mathrm{o}) f(\mathrm{o})=\sum_{w \in \operatorname{Chi}(\mathrm{o})} f^{\prime}(w)+\gamma(\mathrm{o}) f(\mathrm{o})
$$

and hence, since $\left|f^{\prime}(w)\right| \leq\|f\|$ for all $w \in T$, we have

$$
\left|(B f)^{\prime}(\mathrm{o})\right| \leq \gamma(\mathrm{o})\|f\|+\gamma(\mathrm{o})|f(\mathrm{o})| \leq 2 \gamma(\mathrm{o})\|f\| .
$$

Now, for $v \in T^{*}$, we have

$$
\begin{aligned}
(B f)^{\prime}(v) & =\sum_{w \in \operatorname{Chi}(v)} f(w)-\sum_{w \in \operatorname{Chi}(\operatorname{par}(v))} f(w) \\
& =\sum_{w \in \operatorname{Chi}(v)}(f(w)-f(\operatorname{par}(w)))+\gamma(v) f(v)-\sum_{w \in \operatorname{Chi}(\operatorname{par}(v))}(f(w)-f(\operatorname{par}(w)))-\gamma(\operatorname{par}(v)) f(\operatorname{par}(v)) \\
& =\sum_{w \in \operatorname{Chi}(v)} f^{\prime}(w)-\sum_{w \in \operatorname{Chi}(\operatorname{par}(v))} f^{\prime}(w)+\gamma(v) f(v)-\gamma(\operatorname{par}(v)) f(\operatorname{par}(v)),
\end{aligned}
$$

and hence

$$
(B f)^{\prime}(v)=\sum_{w \in \operatorname{Chi}(v)} f^{\prime}(w)-\sum_{w \in \operatorname{Chi}(\operatorname{par}(v))} f^{\prime}(w)+\gamma(v) f^{\prime}(v)+(\gamma(v)-\gamma(\operatorname{par}(v))) f(\operatorname{par}(v))
$$




$$
=\sum_{w \in \operatorname{Chi}(v)} f^{\prime}(w)-\sum_{\substack{w \in \operatorname{Chi}(\operatorname{par}(v)) \\ w \neq v}} f^{\prime}(w)+(\gamma(v)-1) f^{\prime}(v)+(\gamma(v)-\gamma(\operatorname{par}(v))) f(\operatorname{par}(v))
$$

Recalling that $\left|f^{\prime}(v)\right| \leq\|f\|$ for all $v \in T^{*}$, it follows that

$$
\left|(B f)^{\prime}(v)\right| \leq \gamma(v)\|f\|+(\gamma(\operatorname{par}(v))-1)\|f\|+|\gamma(v)-1|\|f\|+|\gamma(v)-\gamma(\operatorname{par}(v))||f(\operatorname{par}(v))| .
$$

Since $|f(\operatorname{par}(v))| \leq(|\operatorname{par}(v)|+1)\|f\|$ by Lemma 2.2, we obtain

$$
\left|(B f)^{\prime}(v)\right| \leq(\gamma(v)+\gamma(\operatorname{par}(v))-1+|\gamma(v)-1|)\|f\|+|\gamma(v)-\gamma(\operatorname{par}(v))||v|\|f\| .
$$

Therefore, $\left|(B f)^{\prime}(v)\right| \leq \Lambda\|f\|$, for all $v \in T^{*}$. Since $\left|(B f)^{\prime}(\mathrm{o})\right| \leq 2 \gamma(\mathrm{o})\|f\|$, it follows that

$$
\|B\| \leq \max \{2 \gamma(\mathrm{o}), \Lambda\},
$$

as desired.

We now provide a more computable estimate of the norm of the backward shift. For a tree $T$, homogeneous by sectors at the level $N$, we define

$$
\Gamma:=\sup \{\gamma(v): v \in T\}=\max \{\gamma(v): 0 \leq|v| \leq N\}
$$

and

$$
\Omega:=\max \{\mid(\gamma(v)-\gamma(\operatorname{par}(v))|| v|: 0<| v \mid \leq N\} .
$$

Observe that if $T$ is homogeneous of order $\gamma$, then $\Gamma=\gamma$ and $\Omega=0$.

The number $\Lambda$ that we defined above can be estimated using $\Gamma$ and $\Omega$. Indeed, if $0<|v| \leq N$ and $\gamma(v) \geq 1$, then

$$
\begin{aligned}
\gamma(v)+\gamma(\operatorname{par}(v))-1+|\gamma(v)-1|+\mid(\gamma(v)-\gamma(\operatorname{par}(v))|| v \mid & =2 \gamma(v)+\gamma(\operatorname{par}(v))-2+\mid(\gamma(v)-\gamma(\operatorname{par}(v))|| v \mid \\
& \leq 3 \Gamma-2+\Omega .
\end{aligned}
$$

If $0<|v| \leq N$ and $\gamma(v)=0$,

$$
\begin{aligned}
\gamma(v)+\gamma(\operatorname{par}(v))-1+|\gamma(v)-1|+\mid(\gamma(v)-\gamma(\operatorname{par}(v))|| v \mid & =\gamma(\operatorname{par}(v))+\mid(\gamma(v)-\gamma(\operatorname{par}(v))|| v \mid \\
& \leq \Gamma+\Omega \\
& \leq 3 \Gamma-2+\Omega .
\end{aligned}
$$

Hence, $\Lambda \leq 3 \Gamma-2+\Omega$ and we obtain the following corollary.

Corollary 4.3. Let $T$ be a rooted, countable infinite and locally finite tree. Assume that $T$ is homogeneous by sectors at the level $N$. Then $B$ is bounded on $\mathcal{L}$. Furthermore,

$$
\|B\| \leq \max \{2 \gamma(\mathrm{o}), 3 \Gamma-2+\Omega\} .
$$

Observe that $3 \Gamma-2+\Omega<2 \gamma(\mathrm{o})$ if and only if $3 \Gamma-2 \gamma(\mathrm{o})+\Omega<2$ and this occurs if and only if $\gamma(\mathrm{o})=\Gamma=1$ and $\Omega=0$. That is, if and only if $T$ is a homogeneous tree of order 1 . We obtain the following corollary.

Corollary 4.4. Let $T$ be a rooted, countable infinite and locally finite tree. Assume that $T$ is homogeneous by sectors at the level $N$. If $T$ is homogeneous of order 1 then $B$ is bounded on $\mathcal{L}$ and $\|B\| \leq 2$. In any other case, $B$ is bounded on $\mathcal{L}$ and $\|B\| \leq 3 \Gamma-2+\Omega$.

The result in the theorem above also holds for the little Lipschitz space.

Theorem 4.5. Let $T$ be a rooted, countable infinite and locally finite tree. Assume that $T$ is homogeneous by sectors at the level $N$. Then $B$ is bounded on $\mathcal{L}_{0}$.

Proof. Since $\mathcal{L}_{0}$ is a closed subspace of $\mathcal{L}$, it is enough to show that, if $f \in \mathcal{L}_{0}$, then $B f \in \mathcal{L}_{0}$.

Let $\epsilon>0$. Since $f \in \mathcal{L}_{0}$, there exists $N_{1}$ such that

$$
\left|f^{\prime}(v)\right|<\frac{\epsilon}{3 \Gamma}, \quad \text { if } \quad|v| \geq N_{1}
$$

By Equation (2), for $v \in T^{*}$ we have

$$
(B f)^{\prime}(v)=\sum_{w \in \operatorname{Chi}(v)} f^{\prime}(w)-\sum_{w \in \operatorname{Chi}(\operatorname{par}(v))} f^{\prime}(w)+\gamma(v) f(v)-\gamma(\operatorname{par}(v)) f(\operatorname{par}(v)) .
$$


Hence, since $\gamma(v)=\gamma(\operatorname{par}(v))$ for every $v \in T^{*}$ with $|v| \geq N+1$, we have that if $|v| \geq \max \left\{N_{1}, N+1\right\}$ then

$$
\begin{aligned}
\left|(B f)^{\prime}(v)\right| & \leq \Gamma \frac{\epsilon}{3 \Gamma}+\Gamma \frac{\epsilon}{3 \Gamma}+|\gamma(v) f(v)-\gamma(\operatorname{par}(v)) f(\operatorname{par}(v))| \\
& \leq \frac{2}{3} \epsilon+\gamma(v)\left|f^{\prime}(v)\right| \\
& \leq \frac{2}{3} \epsilon+\Gamma\left|f^{\prime}(v)\right| \\
& <\epsilon
\end{aligned}
$$

Hence $B f \in \mathcal{L}_{0}$, as desired.

It is clear that the norm of $B$, as an operator on $\mathcal{L}_{0}$, satisfies the same estimates as in Proposition 4.2 and Corollary 4.4 .

We want to prove the converse of Proposition 4.2. For that, we need the following lemma.

Lemma 4.6. Let $T$ be a rooted, countable infinite and locally finite tree. If $\sup \{\gamma(v): v \in T\}=\infty$, then for every $n \in \mathbb{N}$ there exists $v_{n} \in T$ such that $\left|v_{n}\right| \geq n$ and $\gamma\left(\operatorname{par}\left(v_{n}\right)\right)<\gamma\left(v_{n}\right)$.

Proof. By contradiction, assume that there exists $N \in \mathbb{N}$ such that for all $v \in T$ with $|v| \geq N$ we have $\gamma(v) \leq \gamma(\operatorname{par}(v))$. It follows that, for all $k \in \mathbb{N}$, we have that if $|v|=N+k$, then

$$
\gamma(v) \leq \gamma(\operatorname{par}(v)) \leq \gamma\left(\operatorname{par}^{2}(v)\right) \leq \gamma\left(\operatorname{par}^{3}(v)\right) \leq \cdots \leq \gamma\left(\operatorname{par}^{k}(v)\right) .
$$

But, since $\left|\operatorname{par}^{k}(v)\right|=N$, this implies that

$$
\sup \{\gamma(v):|v| \geq N\} \leq \max \{\gamma(u):|u|=N\}
$$

and hence that $\sup \{\gamma(v): v \in T\} \leq \max \{\gamma(v):|v| \leq N\}<\infty$, contradicting the hypothesis.

We can now show that homogeneity by sectors is actually a necessary condition for boundedness of $B$.

Theorem 4.7. Let $T$ be a rooted, countable infinite and locally finite tree. If $B: \mathcal{L} \rightarrow \mathcal{L}$ is bounded, then $T$ is homogeneous by sectors.

Proof. Consider the function $g \in \mathcal{L}$ given by $g(v)=|v|$. Observe that, for every $v \in T^{*}$ we have

$$
\begin{aligned}
(B g)^{\prime}(v) & =\sum_{w \in \operatorname{Chi}(v)}|w|-\sum_{w \in \operatorname{Chi}(\operatorname{par}(v))}|w| \\
& =\gamma(v)(|v|+1)-\gamma(\operatorname{par}(v))(|\operatorname{par}(v)|+1) \\
& =(\gamma(v)-\gamma(\operatorname{par}(v)))|v|+\gamma(v) .
\end{aligned}
$$

First we show that $\sup \{\gamma(v): v \in T\}<\infty$. By contradiction, assume that $\sup \{\gamma(v): v \in T\}=\infty$. By the previous lemma, there exists a sequence $\left(v_{n}\right)$ in $T$ such that $\left|v_{n}\right| \geq n$ and $\gamma\left(\operatorname{par}\left(v_{n}\right)\right)<\gamma\left(v_{n}\right)$. But then, Equation (3) gives

$$
(B g)^{\prime}\left(v_{n}\right)=\left(\gamma\left(v_{n}\right)-\gamma\left(\operatorname{par}\left(v_{n}\right)\right)\right)\left|v_{n}\right|+\gamma\left(v_{n}\right) \geq\left(\gamma\left(v_{n}\right)-\gamma\left(\operatorname{par}\left(v_{n}\right)\right)\right)\left|v_{n}\right| \geq n,
$$

so $(B g)^{\prime}$ is unbounded and hence $B g \notin \mathcal{L}$ contradicting the boundedness of $B$. Hence $\sup \{\gamma(v): v \in T\}<$ $\infty$, as claimed.

Now, let $\Gamma=\sup \{\gamma(v): v \in T\}$. If $T$ is not homogeneous by sectors then, for every $m \in \mathbb{N}$, there exists $v_{m} \in T$ with $\left|v_{m}\right| \geq m$ such that $\gamma\left(v_{m}\right) \neq \gamma\left(\operatorname{par}\left(v_{m}\right)\right)$. But then Equation (3) implies

$$
\begin{aligned}
\left|(B g)^{\prime}\left(v_{m}\right)\right| & =\left|\left(\gamma\left(v_{m}\right)-\gamma\left(\operatorname{par}\left(v_{m}\right)\right)\right)\right| v_{m}\left|+\gamma\left(v_{m}\right)\right| \\
& \geq\left|\gamma\left(v_{m}\right)-\gamma\left(\operatorname{par}\left(v_{m}\right)\right)\right|\left|v_{m}\right|-\left|\gamma\left(v_{m}\right)\right| \\
& \geq\left|v_{m}\right|-\gamma\left(v_{m}\right) \\
& \geq m-\Gamma,
\end{aligned}
$$

which implies that $(B g)^{\prime}$ is unbounded and hence $B g \notin \mathcal{L}$ contradicting the boundedness of $B$. Therefore, $T$ must be homogeneous by sectors.

A similar result holds for $\mathcal{L}_{0}$, with basically the same proof. We include the details for the sake of completeness. 
Theorem 4.8. Let $T$ be a rooted, countable infinite and locally finite tree. If $B: \mathcal{L}_{0} \rightarrow \mathcal{L}_{0}$ is bounded, then $T$ is homogeneous by sectors.

Proof. Consider the function $g \in \mathcal{L}_{0}$ given by $g(v)=\sum_{k=1}^{|v|} \frac{1}{k}$. Observe that, for every $v \in T^{*}$ we have

$$
\begin{aligned}
(B g)^{\prime}(v) & =\sum_{w \in \operatorname{Chi}(v)} \sum_{k=1}^{|w|} \frac{1}{k}-\sum_{w \in \operatorname{Chi}(\operatorname{par}(v))} \sum_{k=1}^{|\operatorname{par}(w)|} \frac{1}{k} \\
& =\gamma(v) \sum_{k=1}^{|v|+1} \frac{1}{k}-\gamma(\operatorname{par}(v)) \sum_{k=1}^{|\operatorname{par}(v)|+1} \frac{1}{k} \\
& =(\gamma(v)-\gamma(\operatorname{par}(v))) \sum_{k=1}^{|v|} \frac{1}{k}+\frac{\gamma(v)}{|v|+1} .
\end{aligned}
$$

First we show that $\sup \{\gamma(v): v \in T\}<\infty$. By contradiction, assume that $\sup \{\gamma(v): v \in T\}=\infty$. By Lemma 4.6, there exists a sequence $\left(v_{n}\right)$ in $T$ such that $\left|v_{n}\right| \geq n$ and $\gamma\left(\operatorname{par}\left(v_{n}\right)\right)<\gamma\left(v_{n}\right)$. But then, Equation (4) gives

$$
(B g)^{\prime}\left(v_{n}\right)=\left(\gamma\left(v_{n}\right)-\gamma\left(\operatorname{par}\left(v_{n}\right)\right)\right) \sum_{k=1}^{\left|v_{n}\right|} \frac{1}{k}+\frac{\gamma\left(v_{n}\right)}{\left|v_{n}\right|+1} \geq\left(\gamma\left(v_{n}\right)-\gamma\left(\operatorname{par}\left(v_{n}\right)\right)\right) \sum_{k=1}^{\left|v_{n}\right|} \frac{1}{k} \geq \sum_{k=1}^{n} \frac{1}{k} .
$$

But this expression is unbounded and so is $(B g)^{\prime}$. Hence $B g \notin \mathcal{L}_{0}$ contradicting the boundedness of $B$.

Now, let $\Gamma=\sup \{\gamma(v): v \in T\}$. If $T$ is not homogeneous by sectors then, for every $m \in \mathbb{N}$, there exists $v_{m} \in T$ with $\left|v_{m}\right| \geq m$ such that $\gamma\left(v_{m}\right) \neq \gamma\left(\operatorname{par}\left(v_{m}\right)\right)$. But then Equation (3) implies

$$
\begin{aligned}
\left|(B g)^{\prime}\left(v_{m}\right)\right| & =\left|\left(\gamma\left(v_{m}\right)-\gamma\left(\operatorname{par}\left(v_{m}\right)\right)\right) \sum_{k=1}^{\left|v_{m}\right|} \frac{1}{k}+\frac{\gamma\left(v_{m}\right)}{\left|v_{n}\right|+1}\right| \\
& \geq\left|\left(\gamma\left(v_{m}\right)-\gamma\left(\operatorname{par}\left(v_{m}\right)\right)\right) \sum_{k=1}^{\left|v_{m}\right|} \frac{1}{k}\right|-\left|\frac{\gamma\left(v_{m}\right)}{\left|v_{m}\right|+1}\right| \\
& \geq \sum_{k=1}^{\left|v_{m}\right|} \frac{1}{k}-\frac{\Gamma}{\left|v_{m}\right|+1} \\
& \geq \sum_{k=1}^{m} \frac{1}{k}-\Gamma
\end{aligned}
$$

which implies that $(B g)^{\prime}$ is unbounded and hence $B g \notin \mathcal{L}_{0}$ contradicting the boundedness of $B$. Therefore, $T$ must be homogeneous by sectors.

The following proposition characterizes trees that are homogeneous by sectors in terms of a combinatorial quantity.

Proposition 4.9. Let $T$ be a rooted, countable infinite and locally finite tree. Then $\sup \{|\gamma(v)-\gamma(\operatorname{par}(v))||v|$ : $\left.v \in T^{*}\right\}<\infty$ if and only if $T$ is homogeneous by sectors.

Proof. First assume that $T$ is homogeneous by sectors at the level $N$. Then, for all $|v|>N$, we have $\gamma(v)=\gamma(\operatorname{par}(v))$ and hence

$$
\left\{|\gamma(v)-\gamma(\operatorname{par}(v))||v|: v \in T^{*}\right\}=\{|\gamma(v)-\gamma(\operatorname{par}(v))||v|: 0<|v| \leq N\} \cup\{0\}
$$

which is a finite set. Hence, $\sup \left\{|\gamma(v)-\gamma(\operatorname{par}(v))||v|: v \in T^{*}\right\}<\infty$.

Now, assume that $\sup \left\{|\gamma(v)-\gamma(\operatorname{par}(v))||v|: v \in T^{*}\right\}<\infty$. If $T$ was not homogeneous by sectors, for every $m \in \mathbb{N}$, there would exist $v_{m} \in T$ with $\left|v_{m}\right| \geq m$ and such that $\gamma\left(v_{m}\right) \neq \gamma\left(\operatorname{par}\left(v_{m}\right)\right)$. But then

$$
\left|\gamma\left(v_{m}\right)-\gamma\left(\operatorname{par}\left(v_{m}\right)\right)\right|\left|v_{m}\right| \geq\left|v_{m}\right| \geq m
$$


and therefore $\sup \left\{|\gamma(v)-\gamma(\operatorname{par}(v))||v|: v \in T^{*}\right\}=\infty$, which is a contradition. Hence $T$ is homogeneous by sectors.

We can summarize the results of this section in the following theorem.

Theorem 4.10. Let $T$ be a rooted, countable infinite and locally finite tree. Let $B$ be the backward shift. The following are equivalent.

- $B: \mathcal{L} \rightarrow \mathcal{L}$ is bounded.

- $B: \mathcal{L}_{0} \rightarrow \mathcal{L}_{0}$ is bounded.

- $T$ is homogeneous by sectors.

- $\sup \left\{|\gamma(v)-\gamma(\operatorname{par}(v))||v|: v \in T^{*}\right\}<\infty$.

Moreover, in this case, $\|B\| \leq \max \{2 \gamma(\mathrm{o}), \Lambda\}$.

We can obtain some simple estimates from below for the norm of the backward shift. Indeed, assume $T$ is homogeneous by sectors and let $g_{1}: T \rightarrow \mathbb{C}$ be defined as (see Figure 3 for an example)

$$
g_{1}(v)= \begin{cases}1, & \text { if } v=\mathrm{o} \\ 2, & \text { if } v \in \mathrm{Chi}(\mathrm{o}) \\ 1, & \text { if } v \in \mathrm{Chi}^{2}(\mathrm{o}) \\ 0, & \text { in any other case }\end{cases}
$$

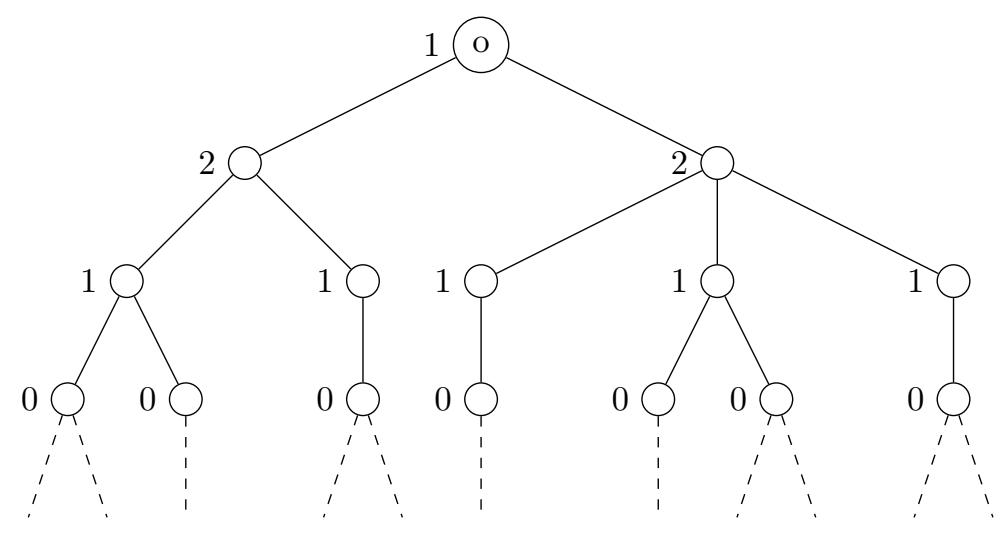

FiguRE 3. The values of the function $g_{1}$ are on the left of each vertex.

Clearly, $g_{1} \in \mathcal{L}$ and $\left\|g_{1}\right\|=1$. But we also have

$$
\left(B g_{1}\right)(v)= \begin{cases}2 \gamma(\mathrm{o}), & \text { if } v=\mathrm{o} \\ \gamma(v), & \text { if } v \in \mathrm{Chi}(\mathrm{o}) \\ 0, & \text { elsewehere }\end{cases}
$$

Then $\left\|B g_{1}\right\| \geq 2 \gamma(\mathrm{o})$ and thus $\|B\| \geq 2 \gamma(\mathrm{o})$. This estimate is part of the next proposition.

Proposition 4.11. Let $T$ be a rooted, countable infinite and locally finite tree. Assume that $T$ is homogeneous by sectors at the level $N$. Then $\|B\| \geq \max \left\{2 \gamma(\mathrm{o}), 3 \Gamma^{\prime}-2\right\}$, where $\Gamma^{\prime}:=\max \{\gamma(v): 0<|v| \leq N\}$.

Proof. Let $u^{*} \in T^{*}$ such that $\gamma\left(u^{*}\right)=\Gamma^{\prime}$. Define the function $g_{2}: T \rightarrow \mathbb{C}$ as (see Figure 4 for an example)

$$
g_{2}(v)= \begin{cases}1, & \text { if } v=\operatorname{par}\left(u^{*}\right), \\ 2, & \text { if } v=u^{*}, \\ 3, & \text { if } v \in \operatorname{Chi}\left(u^{*}\right) \\ 2, & \text { if } v \in \operatorname{Chi}^{2}\left(u^{*}\right), \\ 1, & \text { if } v \in \operatorname{Chi}^{3}\left(u^{*}\right), \\ 0, & \text { in any other case. }\end{cases}
$$




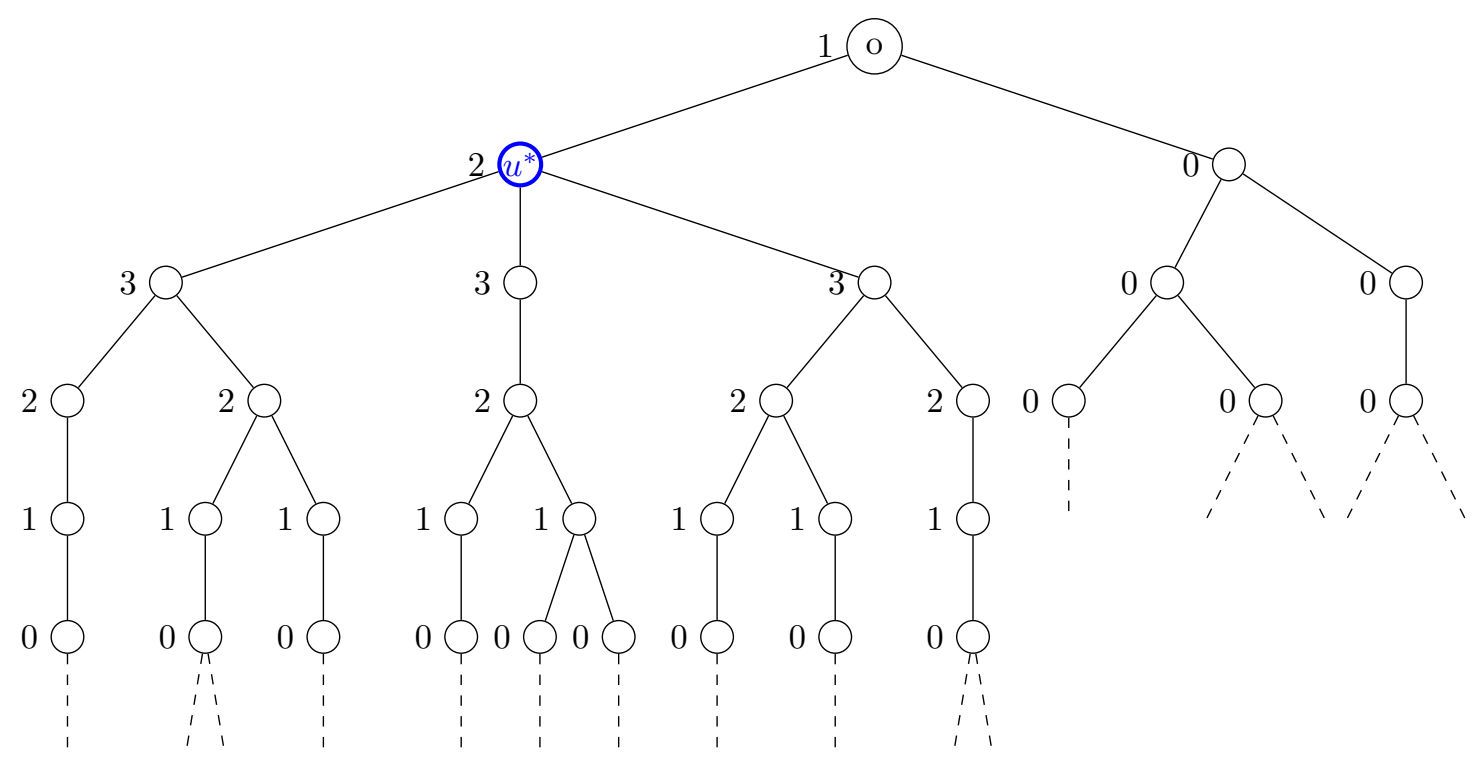

Figure 4. The values of the function $g_{2}$ are on the left of each vertex.

Clearly, $g_{2} \in \mathcal{L}$ and $\left\|g_{2}\right\|=1$. It is easy to check that $\left(B g_{2}\right)\left(u^{*}\right)=3 \gamma\left(u^{*}\right)$ and $\left(B g_{2}\right)\left(\operatorname{par}\left(u^{*}\right)\right)=2$. Hence $\left(B g_{2}\right)^{\prime}\left(u^{*}\right)=3 \gamma\left(u^{*}\right)-2=3 \Gamma^{\prime}-2$. Therefore, $\|B\| \geq 3 \Gamma^{\prime}-2$. By the calculations before the statement of this proposition, it follows that $\|B\| \geq \max \left\{2 \gamma(\mathrm{o}), 3 \Gamma^{\prime}-2\right\}$.

Observe that the same estimate holds for $B$ as operator on $\mathcal{L}_{0}$. For a particular type of trees, the above estimate can be improved.

Proposition 4.12. Let $T$ be a rooted, countable infinite and locally finite tree. Assume that $T$ is homogeneous by sectors at the level $N$. Let $\Gamma^{\prime \prime}:=\max \{\gamma(v):|v|=1\}$. Then $\|B\| \geq \Gamma^{\prime \prime}+2 \gamma(\mathrm{o})-2$.

Proof. Let $u^{*} \in T$, with $\left|u^{*}\right|=1$, be such that $\gamma\left(u^{*}\right)=\Gamma^{\prime \prime}$. Define the function $g_{3}: T \rightarrow \mathbb{C}$ as (see Figure 5 for an example)

$$
g_{3}(v)= \begin{cases}-1, & \text { if } v=\mathrm{o}, \\ 0, & \text { if } v=u^{*}, \\ 1, & \text { if } v \in \mathrm{Chi}\left(u^{*}\right) \\ -2, & \text { if } v \in \mathrm{Chi}(\mathrm{o}), v \neq u^{*} \\ -1, & \text { if } v \in \mathrm{Chi}^{2}(\mathrm{o}), v \notin \operatorname{Chi}\left(u^{*}\right), \\ 0, & \text { in any other case. }\end{cases}
$$

Clearly, $g_{3} \in \mathcal{L}$ and it is straightforward to check that $\left\|g_{3}\right\|=1$. But also, $\left(B g_{3}\right)\left(u^{*}\right)=\gamma\left(u^{*}\right)=\Gamma^{\prime \prime}$ and $\left(B g_{3}\right)\left(\operatorname{par}\left(u^{*}\right)\right)=-2(\gamma(\mathrm{o})-1)$. Hence $\left(B g_{3}\right)^{\prime}\left(u^{*}\right)=\Gamma^{\prime \prime}+2 \gamma(\mathrm{o})-2$. Therefore, $\|B\| \geq \Gamma^{\prime \prime}+2 \gamma(\mathrm{o})-2$, as desired.

It is easily verified that the estimate in Proposition 4.12 is better than the estimate in Proposition 4.11 if $\gamma(\mathrm{o})>\Gamma^{\prime \prime}=\Gamma^{\prime}>2$.

How good are the estimates in Propositions 4.2 and 4.11? In the next section, we will show that for a homogeneous tree, we can find the precise value of $\|B\|$. For now, we show what happens for the case of a tree that is homogeneous by sectors at the level $N=1$.

Proposition 4.13. Let $T$ be a rooted, countable infinite and locally finite tree. Assume that $T$ is homogeneous by sectors at the level 1 . Then

$$
\|B\|=\max \{2 \gamma(\mathrm{o}), \Lambda\},
$$

both as an operator on $\mathcal{L}$ and on $\mathcal{L}_{0}$. 


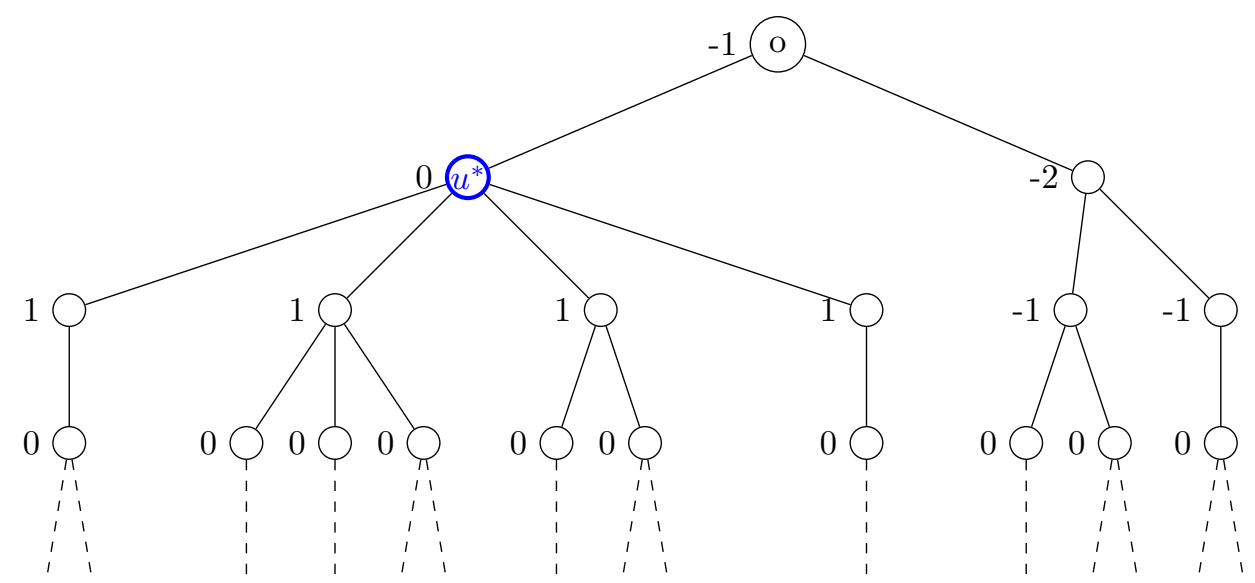

Figure 5. The values of the function $g_{3}$ are on the left of each vertex.

Proof. Let $n=\gamma(\mathrm{o})$ and let $v_{1}, v_{2}, \ldots, v_{n}$ be the children of o. Furthermore, assume that $\gamma\left(v_{1}\right) \leq \gamma\left(v_{2}\right) \leq$ $\cdots \leq \gamma\left(v_{n}\right)$.

Let us deal with the case $\gamma\left(v_{n}\right)=1$ first. It is easy to check that $\Lambda=2 n$ if there exists $j$ with $\gamma\left(v_{j}\right)=0$, and $\Lambda=2 n-1$ if $\gamma\left(v_{j}\right)=1$ for all $j=1,2, \ldots, n$. Hence, by Proposition 4.2, $\|B\| \leq 2 n=2 \gamma(\mathrm{o})$. By proposition 4.11, it follows that $\|B\|=2 \gamma(\mathrm{o})$ and thus $\|B\|=\max \{2 \gamma(\mathrm{o}), \Lambda\}$, as desired.

So assume for the rest of the proof that $\gamma\left(v_{n}\right) \geq 2$. We have two cases:

- Assume $\gamma\left(v_{n}\right) \geq n$. We have three cases:

* If $\gamma\left(v_{j}\right)=0$ for some $j=1,2 \ldots, n-1$, we have that

$$
\begin{aligned}
\gamma\left(v_{j}\right)+\gamma\left(\operatorname{par}\left(v_{j}\right)\right)-1+\left|\gamma\left(v_{j}\right)-1\right|+\left|\gamma\left(v_{j}\right)-\gamma\left(\operatorname{par}\left(v_{j}\right)\right)\right|\left|v_{j}\right| & =2 n \\
& \leq 2 \gamma\left(v_{n}\right) \\
& \leq 3 \gamma\left(v_{n}\right)-2 .
\end{aligned}
$$

* If $1 \leq \gamma\left(v_{j}\right) \leq n$ for some $j=1,2 \ldots, n-1$, we have that

$$
\begin{aligned}
\gamma\left(v_{j}\right)+\gamma\left(\operatorname{par}\left(v_{j}\right)\right)-1+\left|\gamma\left(v_{j}\right)-1\right|+\left|\gamma\left(v_{j}\right)-\gamma\left(\operatorname{par}\left(v_{j}\right)\right)\right|\left|v_{j}\right| & =2 \gamma\left(v_{j}\right)+n-2+\left|\gamma\left(v_{j}\right)-n\right| \\
& =\gamma\left(v_{j}\right)+2 n-2 \\
& \leq 3 \gamma\left(v_{n}\right)-2 .
\end{aligned}
$$

* If $n \leq \gamma\left(v_{j}\right)$ for some $j=1,2 \ldots, n$, we have that

$$
\begin{aligned}
\gamma\left(v_{j}\right)+\gamma\left(\operatorname{par}\left(v_{j}\right)\right)-1+\left|\gamma\left(v_{j}\right)-1\right|+\left|\gamma\left(v_{j}\right)-\gamma\left(\operatorname{par}\left(v_{j}\right)\right)\right|\left|v_{j}\right| & =2 \gamma\left(v_{j}\right)+n-2+\left|\gamma\left(v_{j}\right)-n\right| \\
& =3 \gamma\left(v_{j}\right)-2 \\
& \leq 3 \gamma\left(v_{n}\right)-2 .
\end{aligned}
$$

Thus, $\Lambda=3 \gamma\left(v_{n}\right)-2$, and by Proposition $4.2\|B\| \leq \max \left\{2 n, 3 \gamma\left(v_{n}\right)-2\right\}$. But $2 n \leq 2 \gamma\left(v_{n}\right) \leq$ $3 \gamma\left(v_{n}\right)-2$, since $\gamma\left(v_{n}\right) \geq 2$. Therefore $\|B\| \leq \Lambda=3 \gamma\left(v_{n}\right)-2$. But by Proposition 4.11 we also have $\|B\| \geq 3 \gamma\left(v_{n}\right)-2$, thus $\|B\|=3 \gamma\left(v_{n}\right)-2$. Therefore $\|B\|=\max \{2 \gamma(\mathrm{o}), \Lambda\}$, as desired.

- Assume $n>\gamma\left(v_{n}\right)$.

* If $\gamma\left(v_{j}\right)=0$ for some $j=1,2, \ldots, n-1$, then

$$
\begin{aligned}
\gamma\left(v_{j}\right)+\gamma\left(\operatorname{par}\left(v_{j}\right)\right)-1+\left|\gamma\left(v_{j}\right)-1\right|+\left|\gamma\left(v_{j}\right)-\gamma\left(\operatorname{par}\left(v_{j}\right)\right)\right|\left|v_{j}\right| & =2 n \\
& \leq \gamma\left(v_{n}\right)+2 n-2 .
\end{aligned}
$$

* If $1 \leq \gamma\left(v_{j}\right)$ for some $j=1,2 \ldots, n$, then

$$
\begin{aligned}
\gamma\left(v_{j}\right)+\gamma\left(\operatorname{par}\left(v_{j}\right)\right)-1+\left|\gamma\left(v_{j}\right)-1\right|+\left|\gamma\left(v_{j}\right)-\gamma\left(\operatorname{par}\left(v_{j}\right)\right)\right|\left|v_{j}\right| & =2 \gamma\left(v_{j}\right)+n-2+\left|\gamma\left(v_{j}\right)-n\right| \\
& =\gamma\left(v_{j}\right)+2 n-2 \\
& \leq \gamma\left(v_{n}\right)+2 n-2 .
\end{aligned}
$$


Hence, $\Lambda=\gamma\left(v_{n}\right)+2 n-2$, and by Proposition 4.2. we have $\|B\| \leq \max \left\{2 n, \gamma\left(v_{n}\right)+2 n-2\right\}=$ $\gamma\left(v_{n}\right)+2 n-2$. But now, by Proposition 4.12 we have that $\|B\| \geq \gamma\left(v_{n}\right)+2 n-2$, and therefore $\|B\|=\gamma\left(v_{n}\right)+2 n-2$. Thus $\|B\|=\max \{2 \gamma(\mathrm{o}), \Lambda\}$, as desired.

Are the estimates in Propositions 4.2, 4.11 and 4.12 sharp for $N \geq 2$ ? We have not been able to obtain an answer and we leave open the question of what the norm of $B$ is for general trees.

Note: We would like to thank a referee for suggesting we investigate lower estimates for the norm of $B$, which led to improvements of the estimates we had in a previous version of this paper.

\section{Norm of $B$ and of $B^{n}$ on Homogeneous Trees}

In this section, we find an expression for the norms of $B$ and $B^{n}$ for the case when $T$ is a homogeneous tree. Recall that a tree is homogeneous of order $\gamma$ if $\gamma(v)=\gamma$ for all $v \in T$.

Theorem 5.1. Let $T$ be a rooted homogeneous tree of order $\gamma$ and let $B$ be the backward shift on $\mathcal{L}$. Then

$$
\|B\|= \begin{cases}2, & \text { if } \gamma=1 \\ 3 \gamma-2, & \text { if } \gamma \geq 2 .\end{cases}
$$

Proof. First of all, observe that, by Corollary 4.3 , since $\Gamma=\gamma$ and $\Omega=0$ we have $\|B\| \leq \max \{2 \gamma, 3 \gamma-2\}$.

By Proposition 4.11 we have that $\|B\| \geq \max \{2 \gamma, 3 \gamma-2\}$, and hence the result follows.

We have the same result for the little Lipschitz space.

Theorem 5.2. Let $T$ be a rooted homogeneous tree of order $\gamma$ and let $B$ be the backward shift on $\mathcal{L}_{0}$. Then

$$
\|B\|= \begin{cases}2, & \text { if } \gamma=1, \\ 3 \gamma-2, & \text { if } \gamma \geq 2 .\end{cases}
$$

For the computation of the spectrum of $B$ for a rooted homogeneous tree, we will need to find the norm of $B^{n}$. Let us do some preliminary computations. First of all, it is clear that, for any $f \in \mathcal{F}$ we have

$$
\left(B^{n} f\right)(v)=\sum_{w \in \operatorname{Chi}^{n}(v)} f(w)
$$

From this, and since each vertex in $\mathrm{Chi}^{n-1}(v)$ is the parent of $\gamma$ vertices in $\mathrm{Chi}^{n}(v)$, it follows that

$$
\begin{aligned}
\left(B^{n} f\right)(v) & =\sum_{w \in \mathrm{Chi}^{n}(v)}(f(w)-f(\operatorname{par}(w)))+\sum_{w \in \mathrm{Chi}^{n}(v)} f(\operatorname{par}(w)) \\
& =\sum_{w \in \mathrm{Chi}^{n}(v)} f^{\prime}(w)+\gamma \sum_{w \in \mathrm{Chi}^{n-1}(v)} f(w) .
\end{aligned}
$$

In the same manner, we have

$$
\left(B^{n} f\right)(v)=\sum_{w \in \mathrm{Chi}^{n}(v)} f^{\prime}(w)+\gamma\left(\sum_{w \in \mathrm{Chi}^{n-1}(v)}(f(w)-f(\operatorname{par}(w)))+\gamma \sum_{w \in \mathrm{Chi}^{n-2}(v)} f(w)\right),
$$

since each vertex in $\mathrm{Chi}^{n-2}(v)$ is the parent of $\gamma$ vertices in $\mathrm{Chi}^{n-1}(v)$. Proceeding inductively, we get

$$
\begin{aligned}
\left(B^{n} f\right)(v)= & \sum_{w \in \mathrm{Chi}^{n}(v)} f^{\prime}(w)+\gamma \sum_{w \in \mathrm{Chi}^{n-1}(v)} f^{\prime}(w)+\gamma^{2} \sum_{w \in \mathrm{Chi}^{n-2}(v)} f^{\prime}(w) \\
& +\gamma^{3} \sum_{w \in \mathrm{Chi}^{n-3}(v)} f^{\prime}(w)+\cdots+\gamma^{n-2} \sum_{w \in \mathrm{Chi}^{2}(v)} f^{\prime}(w)+\gamma^{n-1} \sum_{w \in \operatorname{Chi}(v)} f^{\prime}(w)+\gamma^{n} f(v) .
\end{aligned}
$$

In short, we have obtained

$$
\begin{aligned}
\left(B^{n} f\right)(v) & =\sum_{\operatorname{Chi}^{n}(u)} f(w) \\
& =\sum_{k=0}^{n-1} \gamma^{k}\left(\sum_{w \in \operatorname{Chi}^{n-k}(v)} f^{\prime}(w)\right)+\gamma^{n} f(v) .
\end{aligned}
$$


We will use this expression in the proof of the following proposition.

Proposition 5.3. Let $T$ be a rooted homogeneous tree of order $\gamma$ and let $B$ be the backward shift on $\mathcal{L}$. Then,

$$
\left\|B^{n}\right\| \leq \max \left\{(2 n+1) \gamma^{n}-2 n \gamma^{n-1},(n+1) \gamma^{n}\right\}
$$

Proof. Using Equation (6), we have

$$
\left|\left(B^{n} f\right)(\mathrm{o})\right| \leq \sum_{k=0}^{n-1}\left(\gamma^{k} \sum_{w \in \mathrm{Chi}^{n-k}(\mathrm{o})}\left|f^{\prime}(w)\right|\right)+\gamma^{n}|f(\mathrm{o})| .
$$

Since for every $s \in \mathbb{N}$ there are $\gamma^{s}$ vertices in $\mathrm{Chi}^{s}(\mathrm{o})$, and $\left|f^{\prime}(w)\right| \leq\|f\|$ for all $w \in T$, we get

$$
\left|\left(B^{n} f\right)(\mathrm{o})\right| \leq \sum_{k=0}^{n-1} \gamma^{k} \gamma^{n-k}\|f\|+\gamma^{n}|f(\mathrm{o})|,
$$

and therefore, since $|f(\mathrm{o})| \leq\|f\|$, we have

$$
\left|\left(B^{n} f\right)(o)\right| \leq(n+1) \gamma^{n}\|f\| .
$$

Now, let $v \in T^{*}$. Equation (15) is

$$
\left(B^{n} f\right)(v)=\sum_{w \in \mathrm{Chi}^{n}(v)} f^{\prime}(w)+\gamma \sum_{w \in \mathrm{Chi}^{n-1}(v)} f(w) .
$$

Also, we have

$$
\left(B^{n} f\right)(\operatorname{par}(v))=\sum_{w \in \mathrm{Chi}^{n}(\operatorname{par}(v))} f(w)=\sum_{\substack{w \in \mathrm{Chi}^{n}(\operatorname{par}(v)) \\ w \notin \mathrm{Chi}^{n-1}(v)}} f(w)+\sum_{w \in \mathrm{Chi}^{n-1}(v)} f(w) .
$$

The two equations above give

$$
\begin{aligned}
\left(B^{n} f\right)^{\prime}(v) & =\left(B^{n} f\right)(v)-\left(B^{n} f\right)(\operatorname{par}(v)) \\
& =\sum_{w \in \mathrm{Chi}^{n}(v)} f^{\prime}(w)+(\gamma-1) \sum_{w \in \mathrm{Chi}^{n-1}(v)} f(w)-\sum_{\substack{w \in \mathrm{Chi}^{n}(\operatorname{par}(v)) \\
w \notin \mathrm{Chi}^{n-1}(v)}} f(w) .
\end{aligned}
$$

Since each vertex in $\mathrm{Chi}^{n-1}(\operatorname{par}(v))$ is the parent of $\gamma$ vertices in $\mathrm{Chi}^{n}(\operatorname{par}(v))$, we have

$$
\begin{aligned}
& \sum_{\substack{w \in \mathrm{Chi}^{n}(\operatorname{par}(v)) \\
w \notin \mathrm{Chi}^{n-1}(v)}} f(w)=\sum_{\substack{w \in \mathrm{Chi}^{n}(\operatorname{par}(v)) \\
w \notin \mathrm{Chi}^{n-1}(v)}}(f(w)-f(\operatorname{par}(w)))+\sum_{\substack{w \in \mathrm{Chi}^{n}(\operatorname{par}(v)) \\
w \notin \mathrm{Chi}^{n-1}(v)}} f(\operatorname{par}(w)) \\
& =\sum_{\substack{w \in \mathrm{Chi}^{n}(\operatorname{par}(v)) \\
w \notin \mathrm{Chi}^{n-1}(v)}} f^{\prime}(w)+\gamma \sum_{\substack{w \in \mathrm{Chi}^{n-1}(\operatorname{par}(v)) \\
w \notin \mathrm{Chi}^{n-2}(v)}} f(w) .
\end{aligned}
$$

Inductively, we obtain

$$
\begin{aligned}
& \sum_{\substack{w \in \mathrm{Chi}^{n}(\operatorname{par}(v)) \\
w \notin \mathrm{Chi}^{n-1}(v)}} f(w)=\sum_{\substack{w \in \mathrm{Chi}^{n}(\operatorname{par}(v)) \\
w \notin \mathrm{Chi}^{n-1}(v)}} f^{\prime}(w)+\gamma \sum_{\substack{w \in \mathrm{Chi}^{n-1}(\operatorname{par}(v)) \\
w \notin \mathrm{Chi}^{n-2}(v)}} f^{\prime}(w)+\gamma^{2} \sum_{\substack{w \in \mathrm{Chi}^{n-2}(\operatorname{par}(v)) \\
w \notin \mathrm{Chi}^{n-3}(v)}} f^{\prime}(w) \\
& +\cdots+\gamma^{n-2} \sum_{\substack{w \in \mathrm{Chi}^{2}(\operatorname{par}(v)) \\
w \notin \operatorname{Chi}(\operatorname{par}(v))}} f^{\prime}(w)+\gamma^{n-1} \sum_{\substack{w \in \operatorname{Chi}(\operatorname{par}(v)) \\
w \neq v}} f(w) .
\end{aligned}
$$

Since there are $\gamma-1$ vertices in $\operatorname{Chi}(\operatorname{par}(v))$ different than $v$, we have

$$
\begin{aligned}
& \sum_{\substack{w \in \mathrm{Chi}^{n}(\operatorname{par}(v)) \\
w \notin \mathrm{Chi}^{n-1}(v)}} f(w)=\sum_{\substack{w \in \mathrm{Chi}^{n}(\operatorname{par}(v)) \\
w \notin \mathrm{Chi}^{n-1}(v)}} f^{\prime}(w)+\gamma \sum_{\substack{w \in \mathrm{Chi}^{n-1}(\operatorname{par}(v)) \\
w \notin \mathrm{Chi}^{n-2}(v)}} f^{\prime}(w)+\gamma^{2} \sum_{\substack{w \in \mathrm{Chi}^{n-2}(\operatorname{par}(v)) \\
w \notin \mathrm{Chi}^{n-3}(v)}} f^{\prime}(w) \\
& +\cdots+\gamma^{n-2} \sum_{\substack{w \in \mathrm{Chi}^{2}(\operatorname{par}(v)) \\
w \notin \operatorname{Chi}(\operatorname{par}(v))}} f^{\prime}(w)+\gamma^{n-1} \sum_{\substack{w \in \operatorname{Chi}(\operatorname{par}(v)) \\
w \neq v}} f^{\prime}(w)+\gamma^{n-1}(\gamma-1) f(\operatorname{par}(v)) .
\end{aligned}
$$


In short, we have obtained

$$
\sum_{\substack{w \in \mathrm{Chi}^{n}(\operatorname{par}(v)) \\ w \notin \mathrm{Chi}^{n-1}(v)}} f(w)=\sum_{k=0}^{n-1} \gamma^{k}\left(\sum_{\substack{w \in \mathrm{Chi}^{n-k}(\operatorname{par}(v)) \\ w \notin \mathrm{Chi}^{n-k-1}(v)}} f^{\prime}(w)\right)+\gamma^{n-1}(\gamma-1) f(\operatorname{par}(v)) .
$$

Substituting Equation (6) (for $B^{n-1}$ ) and (8) into Equation (7) we obtain

$$
\begin{aligned}
& \left(B^{n} f\right)^{\prime}(v)=\left(B^{n} f\right)(v)-\left(B^{n} f\right)(\operatorname{par}(v)) \\
& =\sum_{w \in \mathrm{Chi}^{n}(v)} f^{\prime}(w)+(\gamma-1)\left(\sum_{k=0}^{n-2} \gamma^{k}\left(\sum_{w \in \mathrm{Chi}^{n-k-1}(v)} f^{\prime}(w)\right)+\gamma^{n-1} f(v)\right) \\
& -\sum_{k=0}^{n-1} \gamma^{k}\left(\sum_{\substack{w \in \mathrm{Chi}^{n-k}(\operatorname{par}(v)) \\
w \notin \mathrm{Chi}^{n-k-1}(v)}} f^{\prime}(w)\right)-\gamma^{n-1}(\gamma-1) f(\operatorname{par}(v)) \\
& =\sum_{w \in \mathrm{Chi}^{n}(v)} f^{\prime}(w)+(\gamma-1)\left(\sum_{k=0}^{n-2} \gamma^{k}\left(\sum_{w \in \mathrm{Chi}^{n-k-1}(v)} f^{\prime}(w)\right)\right) \\
& -\sum_{k=0}^{n-1} \gamma^{k}\left(\sum_{\substack{w \in \mathrm{Chi}^{n-k}(\operatorname{par}(v)) \\
w \notin \mathrm{Chi}^{n-k-1}(v)}} f^{\prime}(w)\right)+\gamma^{n-1}(\gamma-1) f^{\prime}(v)
\end{aligned}
$$

Since, for every $s \in \mathbb{N}$, there are $\gamma^{s}$ vertices in $\operatorname{Chi}^{s}(v)$, and for every $k \in\{1,2, \ldots, n-1\}$ there are $\gamma^{n-k}-\gamma^{n-k-1}$ vertices in $\mathrm{Chi}^{n-k}(\operatorname{par}(v)) \backslash \mathrm{Chi}^{n-k-1}(v)$, we obtain

$$
\begin{aligned}
\left.\mid\left(B^{n} f\right)^{\prime}(v)\right) \mid & \leq \gamma^{n}\|f\|+(\gamma-1) \sum_{k=0}^{n-2} \gamma^{k} \gamma^{n-k-1}\|f\|+\sum_{k=0}^{n-1} \gamma^{k}\left(\gamma^{n-k}-\gamma^{n-k-1}\right)\|f\|+\gamma^{n-1}(\gamma-1)\|f\| \\
& =\left(\gamma^{n}+(\gamma-1) \sum_{k=0}^{n-2} \gamma^{n-1}+\sum_{k=0}^{n-1}\left(\gamma^{n}-\gamma^{n-1}\right)+\gamma^{n-1}(\gamma-1)\right)\|f\| \\
& =\left(\gamma^{n}+(n-1)(\gamma-1) \gamma^{n-1}+n\left(\gamma^{n}-\gamma^{n-1}\right)+\gamma^{n-1}(\gamma-1)\right)\|f\| \\
& =\left((2 n+1) \gamma^{n}-2 n \gamma^{n-1}\right)\|f\| .
\end{aligned}
$$

Therefore, $\left\|B^{n}\right\| \leq \max \left\{(2 n+1) \gamma^{n}-2 n \gamma^{n-1},(n+1) \gamma^{n}\right\}$, as desired.

Using this proposition we can compute the exact value of the norm of $B^{n}$.

Theorem 5.4. Let $T$ be a rooted homogeneous tree of order $\gamma$ and let $B$ be the backward shift on $\mathcal{L}$. Then, for every $n \in \mathbb{N}$,

$$
\left\|B^{n}\right\|= \begin{cases}n+1, & \text { if } \gamma=1 \\ (2 n+1) \gamma^{n}-2 n \gamma^{n-1}, & \text { if } \gamma \geq 2 .\end{cases}
$$

Proof. First of all, observe that, by Proposition 5.3 we have $\|B\| \leq \max \left\{(2 n+1) \gamma^{n}-2 n \gamma^{n-1}, n+1\right\}$, which equals $n+1$ if $\gamma=1$, and $(2 n+1) \gamma^{n}-2 n \gamma^{n-1}$ if $\gamma \geq 2$. 
Choose a fixed $u^{*} \in \operatorname{Chi}(\mathrm{o})$. Define the function $h_{n}: T \rightarrow \mathbb{C}$ (see Figure 6 for an example) as

$$
h_{n}(v)= \begin{cases}1, & \text { if } v=0, \\ |v|+1, & \text { if } v \in S_{u^{*}} \text { and } 1 \leq|v| \leq n+1, \\ (2 n+3)-|v|, & \text { if } v \in S_{u^{*}} \text { and } n+1 \leq|v| \leq 2 n+3, \\ -|v|+1, & \text { if } v \notin S_{u^{*}} \text { and } 1 \leq|v| \leq n, \\ -(2 n-1)+|v|, & \text { if } v \notin S_{u^{*}} \text { and } n \leq|v| \leq 2 n-1, \\ 0, & \text { in any other case. }\end{cases}
$$

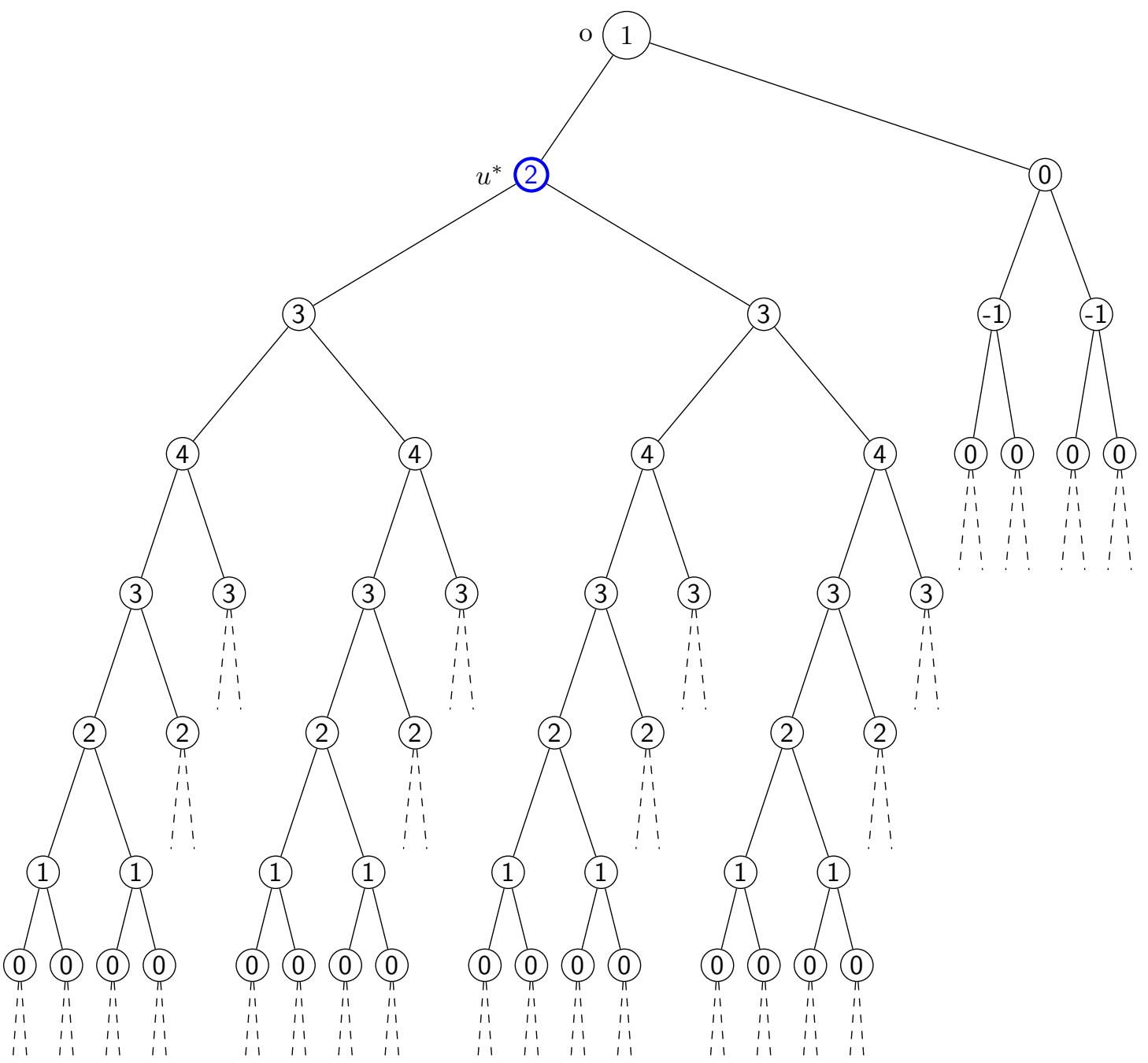

Figure 6. Some of the values of $h_{n}$ for the case $\gamma=2$ and $n=2$. The values are inside each vertex, the blue vertex is $u^{*}$ and the root is labeled $o$.

It is clear that

$$
h_{n}^{\prime}(v)= \begin{cases}1, & \text { if } v=0 \\ 1, & \text { if } v \in S_{u^{*}} \text { and } 1 \leq|v| \leq n+1 \\ -1, & \text { if } v \in S_{u^{*}} \text { and } n+2 \leq|v| \leq 2 n+3 \\ -1, & \text { if } v \notin S_{u^{*}} \text { and } 1 \leq|v| \leq n \\ 1, & \text { if } v \notin S_{u^{*}} \text { and } n+1 \leq|v| \leq 2 n-1 \\ 0, & \text { in any other case. }\end{cases}
$$


and therefore $h_{n} \in \mathcal{L}$ and $\left\|h_{n}\right\|=1$. Also, a straightforward computation shows that

$$
\left(B^{n} h_{n}\right)(v)= \begin{cases}\gamma^{n-1}(n+1)+\left(\gamma^{n}-\gamma^{n-1}\right)(-n+1), & \text { if } v=0, \\ \gamma^{n}(n+3-|v|), & \text { if } v \in S_{u^{*}} \text { and } 1 \leq|v| \leq n+3, \\ \gamma^{n}(-(n-1)+|v|), & \text { if } v \notin S_{u^{*}} \text { and } 1 \leq|v| \leq n-1, \\ 0, & \text { in any other case. }\end{cases}
$$

Hence,

(9)

$$
\left(B^{n} h_{n}\right)^{\prime}(v)= \begin{cases}\gamma^{n-1}(n+1)+\left(\gamma^{n}-\gamma^{n-1}\right)(-n+1), & \text { if } v=\mathrm{o}, \\ \gamma^{n}(n+2)-\left(\gamma^{n-1}(n+1)+\left(\gamma^{n}-\gamma^{n-1}\right)(-n+1)\right), & \text { if } v=u^{*}, \\ \gamma^{n}(-n+2)-\left(\gamma^{n-1}(n+1)+\left(\gamma^{n}-\gamma^{n-1}\right)(-n+1)\right), & \text { if } v \neq u^{*} \text { and }|v|=1, \\ -\gamma^{n}, & \text { if } v \in S_{u^{*}} \text { and } 2 \leq|v| \leq n+3, \\ \gamma^{n}, & \text { if } v \notin S_{u^{*}} \text { and } 2 \leq|v| \leq n-1, \\ 0, & \text { in any other case. }\end{cases}
$$

If $\gamma=1$, Equation (9) simplifies to

$$
\left(B^{n} h_{n}\right)^{\prime}(v)= \begin{cases}n+1, & \text { if } v=0 \\ 1, & \text { if } v=u^{*} \\ -1, & \text { if } 2 \leq|v| \leq n+3 \\ 0, & \text { in any other case. }\end{cases}
$$

Hence, if $\gamma=1$, we have then that $\left\|B^{n} h_{n}\right\|=n+1$, which together with Proposition 5.3 gives that $\left\|B^{n}\right\|=n+1$, as desired.

If $\gamma \geq 2$, Equation (9) simplifies to

$$
\left(B^{n} h_{n}\right)^{\prime}(v)= \begin{cases}\gamma^{n-1}(2 n)-\gamma^{n}(n-1), & \text { if } v=0, \\ \gamma^{n}(2 n+1)-\gamma^{n-1}(2 n), & \text { if } v=u^{*}, \\ \gamma^{n}-\gamma^{n-1}(2 n), & \text { if } v \neq u^{*} \text { and }|v|=1, \\ -\gamma^{n}, & \text { if } v \in S_{u^{*}} \text { and } 2 \leq|v| \leq n+3, \\ \gamma^{n}, & \text { if } v \notin S_{u^{*}} \text { and } 2 \leq|v| \leq n-1, \\ 0, & \text { in any other case. }\end{cases}
$$

It can be checked that

$$
\max \left\{\left|\gamma^{n-1}(2 n)-\gamma^{n}(n-1)\right|,\left|\gamma^{n}(2 n+1)-\gamma^{n-1}(2 n)\right|,\left|\gamma^{n}-\gamma^{n-1}(2 n)\right|,\left|\gamma^{n}\right|\right\}=\gamma^{n}(2 n+1)-\gamma^{n-1}(2 n)
$$

and hence $\left\|B^{n} h_{n}\right\|=\gamma^{n}(2 n+1)-\gamma^{n-1}(2 n)$, which together with Proposition 5.3 gives

$$
\left\|B^{n}\right\|=\gamma^{n}(2 n+1)-\gamma^{n-1}(2 n),
$$

as desired.

Observe that, in the previous proof, the function $h_{n}$ is also in $\mathcal{L}_{0}$. Hence we also obtain

Theorem 5.5. Let $T$ be a rooted homogeneous tree of order $\gamma$ and let $B$ be the backward shift on $\mathcal{L}_{0}$. Then

$$
\left\|B^{n}\right\|= \begin{cases}n+1, & \text { if } \gamma=1 \\ (2 n+1) \gamma^{n}-(2 n) \gamma^{n-1}, & \text { if } \gamma \geq 2 .\end{cases}
$$

\section{Spectrum of $B$ on Homogeneous Trees}

In this section, we compute the spectrum of $B$ for both the Lipschitz and the little Lipschitz space in the case where $T$ is a rooted homogeneous tree. First, we obtain part of the set of eigenvalues. We will show later that we actually have an equality. 
Theorem 6.1. Let $T$ be a rooted homogeneous tree of order $\gamma$. If $B$ is the backward shift on $\mathcal{L}$, then

$$
\{\lambda \in \mathbb{C}:|\lambda| \leq \gamma\} \subseteq \sigma_{\mathrm{p}}(B) .
$$

If $B$ is the backward shift on $\mathcal{L}_{0}$, then

$$
\{\lambda \in \mathbb{C}:|\lambda|<\gamma\} \cup\{\gamma\} \subseteq \sigma_{\mathrm{p}}(B) .
$$

Proof. Define $f_{\lambda} \in \mathcal{F}$ as $f_{\lambda}(v)=(\lambda / \gamma)^{|v|}$. Then,

$$
\left(B f_{\lambda}\right)(v)=\sum_{w \in \operatorname{Chi}(v)} f_{\lambda}(w)=\sum_{w \in \operatorname{Chi}(v)}(\lambda / \gamma)^{|w|}=\gamma(\lambda / \gamma)^{|v|+1}=\left(\lambda f_{\lambda}\right)(v) .
$$

For $v \in T^{*}$ we have

$$
f_{\lambda}^{\prime}(v)=(\lambda / \gamma)^{|v|}-(\lambda / \gamma)^{|\operatorname{par}(v)|}=(\lambda / \gamma)^{|v|-1}(\lambda / \gamma-1) .
$$

Hence, $f_{\lambda} \in \mathcal{L}$ if and only if $|\lambda| \leq \gamma$ and $f_{\lambda} \in \mathcal{L}_{0}$ if and only if $|\lambda|<\gamma$ or $\lambda=\gamma$. The result now follows immediately.

With this, we are ready to prove the following theorem.

Theorem 6.2. Let $T$ be a rooted homogeneous tree of order $\gamma$. If $B$ is the backward shift on $\mathcal{L}$, then

$$
\sigma(B)=\sigma_{\mathrm{ap}}(B)=\sigma_{\mathrm{p}}(B)=\{\lambda \in \mathbb{C}:|\lambda| \leq \gamma\} .
$$

Proof. First, we will compute the spectral radius of $B$. By Theorem 5.4 if $\gamma=1$ then

$$
r(B)=\lim _{n \rightarrow \infty}\left\|B^{n}\right\|^{1 / n}=\lim _{n \rightarrow \infty}(n+1)^{1 / n}=1=\gamma .
$$

If $\gamma \geq 2$, Theorem 5.4 gives

$$
r(B)=\lim _{n \rightarrow \infty}\left\|B^{n}\right\|^{1 / n}=\lim _{n \rightarrow \infty}\left((2 n+1) \gamma^{n}-(2 n) \gamma^{n-1}\right)^{1 / n}=\lim _{n \rightarrow \infty} \gamma^{(n-1) / n}((2 n+1) \gamma-2 n)^{1 / n}=\gamma .
$$

Therefore, $\sigma(B) \subseteq\{\lambda \in \mathbb{C}:|\lambda| \leq \gamma\}$. This and the previous theorem imply that

$$
\{\lambda \in \mathbb{C}:|\lambda| \leq \gamma\} \subseteq \sigma_{\mathrm{p}}(B) \subseteq \sigma_{\mathrm{ap}}(B) \subseteq \sigma(B) \subseteq\{\lambda \in \mathbb{C}:|\lambda| \leq \gamma\},
$$

and hence the result follows.

We obtain a similar result for the backward shift on $\mathcal{L}_{0}$.

Theorem 6.3. Let $T$ be a rooted homogeneous tree of order $\gamma$. If $B$ is the backward shift on $\mathcal{L}_{0}$, then

$$
\sigma(B)=\sigma_{\mathrm{ap}}(B)=\{\lambda \in \mathbb{C}:|\lambda| \leq \gamma\} .
$$

Proof. As was the case in the theorem above, by Theorem 5.5 we have $r(B)=\gamma$ and hence

$$
\sigma(B) \subseteq\{\lambda \in \mathbb{C}:|\lambda| \leq \gamma\} .
$$

This, and Theorem 6.1 give that

$$
\sigma(B)=\{\lambda \in \mathbb{C}:|\lambda| \leq \gamma\}
$$

Since, for any operator $A$ we have $\partial \sigma(A) \subseteq \sigma_{a p}(A)$ (see, e.g. [15, p. 210]), we have that

$$
\{\lambda \in \mathbb{C}:|\lambda|=\gamma\} \subseteq \sigma_{a p}(B),
$$

and, again, by Theorem 6.1, we obtain

$$
\sigma_{a p}(B)=\{\lambda \in \mathbb{C}:|\lambda| \leq \gamma\},
$$

which completes the proof.

With the previous result showing what the spectrum of the backward shift is, we can determine the point spectrum.

Theorem 6.4. Let $T$ be a rooted homogeneous tree of order $\gamma$. If $B$ is the backward shift on $\mathcal{L}_{0}$, then

$$
\sigma_{\mathrm{p}}(B)=\{\lambda \in \mathbb{C}:|\lambda|<\gamma\} \cup\{\gamma\} .
$$


Proof. By Theorems 6.1 and 6.3 it suffices to show that if $\lambda \neq \gamma$ and $|\lambda|=\gamma$, then $\lambda \notin \sigma_{p}(B)$. So let $\lambda \neq \gamma$ with $|\lambda|=\gamma$ and assume then that $B f=\lambda f$ for a nonzero $f \in \mathcal{L}_{0}$.

First, since $f$ is not zero, there exists a vertex $w^{*}$ such that $f\left(w^{*}\right) \neq 0$. Dividing by a constant, if necessary, we may assume that $f\left(w^{*}\right)=1$

Now, we claim that for all $n \in \mathbb{N}$ there exists $v \in \mathrm{Chi}^{n}\left(w^{*}\right)$ with $|f(v)| \geq 1$. Indeed, suppose this was not the case. Then, for some $m \in \mathbb{N}$ we would have $|f(v)|<1$ for all $v \in \mathrm{Chi}^{m}\left(w^{*}\right)$. But since $B^{m} f=\lambda^{m} f$, we have

$$
\lambda^{m} f\left(w^{*}\right)=\sum_{v \in \operatorname{Chi}^{m}\left(w^{*}\right)} f(v)
$$

and hence we obtain

$$
|\lambda|^{m}=\left|\lambda^{m} f\left(w^{*}\right)\right| \leq \sum_{v \in \operatorname{Chi}^{m}\left(w^{*}\right)}|f(v)|<\gamma^{m} 1=|\lambda|^{m},
$$

which is a contradiction, so the claim is true.

Now, since $f \in \mathcal{L}_{0}$, there exists $N \in \mathbb{N}$ such that, for all $|v| \geq N$ we have

$$
\left|f^{\prime}(v)\right|=|f(v)-f(\operatorname{par}(v))|<\frac{|\gamma-\lambda|}{2 \gamma}
$$

By the claim, there exists $u^{*} \in \mathrm{Chi}^{N}\left(w^{*}\right)$ with $\left|f\left(u^{*}\right)\right| \geq 1$. Hence

$$
\sum_{u \in \operatorname{Chi}\left(u^{*}\right)}(f(u)-f(\operatorname{par}(u)))=(B f)\left(u^{*}\right)-\gamma f\left(u^{*}\right)=(\lambda-\gamma) f\left(u^{*}\right) .
$$

But then,

$$
|\lambda-\gamma|\left|f\left(u^{*}\right)\right| \leq \sum_{u \in \operatorname{Chi}\left(u^{*}\right)}|f(u)-f(\operatorname{par}(u))|<\gamma \frac{|\gamma-\lambda|}{2 \gamma},
$$

since every $u \in \operatorname{Chi}\left(u^{*}\right)$ satisfies $|u|>N$. But the last display implies that $\left|f\left(u^{*}\right)\right|<\frac{1}{2}$, which is a contradiction. Hence there cannot be $\lambda \neq \gamma$ with $|\lambda|=\gamma$ and $B f=\lambda f$ for a nonzero $f \in \mathcal{L}_{0}$, which completes the proof of the theorem.

\section{HYPERCYCLICITY}

In [13, it is shown that $\mathcal{L}$ (with an equivalent norm) is not separable, while $\mathcal{L}_{0}$ is separable (this was originally shown in [11]). So, in order to study hypercyclicity of operators, we need to restrict ourselves to $\mathcal{L}_{0}$, which we do from now on.

First, we get rid of the question of whether $S$ is hypercyclic. It is not since the norm of $S$ is one and therefore $S$ can never be hypercyclic. We offer an alternative proof.

Theorem 7.1. Let $T$ be a rooted, countably infinite and locally finite tree and let $S$ be the forward shift on $\mathcal{L}_{0}$. Then $S$ is not hypercyclic.

Proof. If $S$ were hypercyclic, then there would exist $f \in \mathcal{L}_{0}$ and a natural number $N$ such that

$$
\left\|S^{N} f-\chi_{\{\circ\}}\right\|<\frac{1}{2}
$$

where $\chi_{\{\mathrm{o}\}}$ is the characteristic function of the root o. The definition of the norm in $\mathcal{L}$ then would imply that

$$
\left|\left(S^{N} f\right)(\mathrm{o})-1\right| \leq\left\|S^{N} f-\chi_{\{\mathrm{o}\}}\right\|<\frac{1}{2} .
$$

But, since $\left(S^{n} f\right)(\mathrm{o})=0$ for every $n \in \mathbb{N}$, this is a contradiction. Therefore $S$ is not hypercyclic.

We will use the following lemma, which is proved in [14].

Lemma 7.2. Let $X$ be the set of all functions in $\mathcal{L}_{0}$ with finite support. Then $X$ is dense in $\mathcal{L}_{0}$.

The following definition will be useful to characterize hypercyclicity.

Definition 7.3. Let $T$ a rooted tree and $v \in T$. We say that $S_{v}$ is a free end (at $v$ ) if for all $w \in S_{v}$ we have $\gamma(w)=1$. 


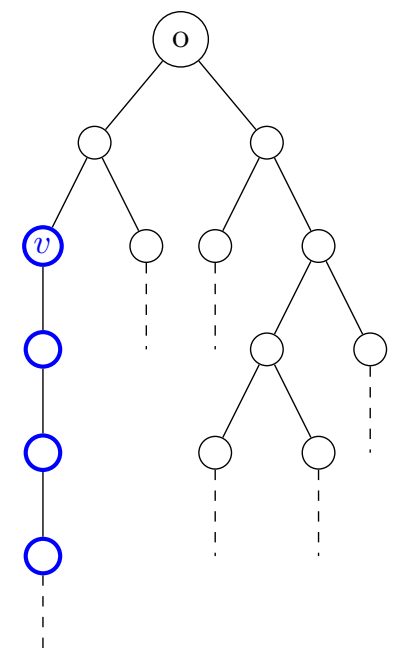

FiguRE 7. Free end starting at the vertex $v$.

Recall that $T^{n}$ denotes the set of vertices that have $n$-parents; i.e., $v \in T^{n}$ if there exists $u \in T$ with $v \in \mathrm{Chi}^{n}(u)$. Also, recall that $\gamma(u, n)$ denotes the number of vertices in the set $\mathrm{Chi}^{n}(u)$.

We define the function $\beta: T \times \mathbb{N} \rightarrow \mathbb{R}$ as

$$
\beta(v, n)= \begin{cases}\frac{1}{\gamma\left(\operatorname{par}^{n}(v), n\right)}, & \text { if } v \in T^{n}, \\ 0, & \text { if } v \notin T^{n} .\end{cases}
$$

The following lemma will be used later.

Lemma 7.4. Let $T$ be a rooted, countably infinite and locally finite tree. If $T$ is homogeneous by sectors and has no free ends then

$$
\sup _{w \in T} \beta(w, n) \rightarrow 0 \quad \text { as } n \rightarrow \infty
$$

Proof. Since $T$ is homogeneous by sectors, there exists $M \in \mathbb{N}$ such that for every $v \in T$ with $|v|=M$, we have $\gamma(v)=\gamma(u)$ for every $u \in S_{v}$. Since $T$ is locally finite, there exist finitely many such $v$, say $v_{1}, v_{2}, \ldots, v_{r}$. For each $j=1,2, \ldots, r$, define $\mu_{j}:=\gamma\left(v_{j}\right)$. Since $T$ has no free ends, $\mu:=\min \left\{\mu_{1}, \mu_{2}, \ldots, \mu_{r}\right\} \geq 2$.

Let $w \in T$ and let $n \geq 2 M$.

- If $|w|<n$, then $w \notin T^{n}$ and hence

$$
\beta(w, n)=0 .
$$

- If $|w| \geq M+n$, then $\left|\operatorname{par}^{n}(w)\right| \geq M$ and hence $\gamma\left(\operatorname{par}^{n}(w), n\right) \geq \mu^{n}$. Therefore,

$$
\beta(w, n) \leq \frac{1}{\mu^{n}} .
$$

- If $n \leq|w|<M+n$, let $k=|w|$. Then, since $k-M<n$, we have $\mathrm{Chi}^{k-M}\left(\operatorname{par}^{k-M}(w)\right) \subseteq$ $\mathrm{Chi}^{n}\left(\operatorname{par}^{n}(w)\right)$ and hence

$$
\gamma\left(\operatorname{par}^{k-M}(w), k-M\right) \leq \gamma\left(\operatorname{par}^{n}(w), n\right)
$$

But clearly $\left|\operatorname{par}^{k-M}(w)\right|=M$ which implies that $\gamma\left(\operatorname{par}^{k-M}(w), k-M\right) \geq \mu^{k-M}$ and hence $\beta(w, n) \leq$ $\frac{1}{\mu^{k-M}}$. Since $k>n$ this gives

$$
\beta(w, n) \leq \frac{1}{\mu^{n-M}}
$$

Hence, for every $w \in T$, we have

$$
\beta(w, n) \leq \frac{1}{\mu^{n-M}}
$$


if $n>2 M$. Therefore, if $n>2 M$, then

$$
\sup _{w \in T} \beta(w, n) \leq \frac{1}{\mu^{n-M}}
$$

and thus

$$
\sup _{v \in T} \beta(v, n) \rightarrow 0 \quad \text { as } n \rightarrow \infty
$$

We can now give a sufficient condition for hypercyclicity of $B$.

Theorem 7.5. Let $T$ be an countably infinite and locally finite tree and assume that $B$ is bounded on $\mathcal{L}_{0}$. If $T$ has no free ends, then $B$ is hypercyclic.

Proof. To show hypercyclicity of $B$, we will use the Hypercyclicity Criterion (Theorem 2.3). Let $X$ be the set of all functions with finite support and for each $n \in \mathbb{N}$, define the function $R_{n}: X \rightarrow \mathcal{L}$ as

$$
\left(R_{n} f\right)(v)= \begin{cases}\beta(v, n) f\left(\operatorname{par}^{n}(v)\right), & \text { if } v \in T^{n}, \text { and } \\ 0, & \text { if } v \notin T^{n} .\end{cases}
$$

(Observe that $R_{n} f$ also has finite support.)

(1) First, let $f \in X$. Choose $N \in \mathbb{N}$ such that $f(v)=0$ for all $v$ with $|v| \geq N$. Then, for all $v \in T$,

$$
\left(B^{n} f\right)(v)=\sum_{w \in \operatorname{Chi}^{n}(v)} f(w)=0
$$

if $n>N$ and, similarly, for all $v \in T^{*}$ we have $\left(B^{n} f\right)(\operatorname{par}(v))=0$ if $n>N$. Hence $\left(B^{n} f\right)^{\prime}=0$ as long as $n>N$. Therefore $B^{n} f \rightarrow 0$, as $n \rightarrow \infty$, as desired.

(2) Let $f \in X$ and $v \in T$. Since $f$ is of finite support, there exists $M>0$ such that $|f(v)| \leq M$ for all $v \in T$.

- If $|v|<n$, then $\left(R_{n} f\right)(v)=0$; while if $0<|v|<n$, then $\left(R_{n} f\right)(\operatorname{par}(v))=0$. Hence $\left(R_{n} f\right)^{\prime}(v)=$ 0 if $|v|<n$.

- If $|v|=n$, then, since $\operatorname{par}(v) \notin T^{n}$, then

$$
\begin{aligned}
\left|\left(R_{n} f\right)^{\prime}(v)\right| & =\left|\left(R_{n} f\right)(v)-\left(R_{n} f\right)(\operatorname{par}(v))\right| \\
& =\beta(v, n)\left|f\left(\operatorname{par}^{n}(v)\right)\right| \\
& \leq M \beta(v, n) .
\end{aligned}
$$

- If $|v|>n$. Then,

$$
\begin{aligned}
\left|\left(R_{n} f\right)^{\prime}(v)\right| & =\left|\left(R_{n} f\right)(v)-\left(R_{n} f\right)(\operatorname{par}(v))\right| \\
& \leq \beta(v, n)\left|f\left(\operatorname{par}^{n}(v)\right)\right|+\beta(\operatorname{par}(v), n)\left|f\left(\operatorname{par}^{n+1}(v)\right)\right| \\
& \leq M(\beta(v, n)+\beta(\operatorname{par}(v), n))
\end{aligned}
$$

Therefore, for all $v \in T$, we have

$$
\left|\left(R_{n} f\right)^{\prime}(v)\right| \leq 2 M \sup _{v \in T} \beta(v, n)
$$

and hence

$$
\left\|R_{n} f\right\| \leq 2 M \sup _{v \in T} \beta(v, n) .
$$

Therefore, since $T$ has no free ends, by Lemma 7.4 (since $B$ is bounded and hence $T$ is homogeneous by sectors) we have that $R_{n} f \rightarrow 0$, as desired. 
(3) Now, let $v \in T$. We then have

$$
\begin{aligned}
\left(B^{n}\left(R_{n} f\right)\right)(v) & =\sum_{w \in \mathrm{Chi}^{n}(v)}\left(R_{n} f\right)(w) \\
& =\sum_{w \in \mathrm{Chi}^{n}(v)} \frac{1}{\gamma\left(\operatorname{par}^{n}(w), n\right)} f\left(\operatorname{par}^{n}(w)\right) \\
& =\sum_{w \in \mathrm{Chi}^{n}(v)} \frac{1}{\gamma(v, n)} f(v) \\
& =f(v) .
\end{aligned}
$$

Therefore, $B^{n} R_{n} f \rightarrow f$ as $n \rightarrow \infty$, as desired.

Since all conditions in the hyperciclicity criterion hold, it follows that $B$ is hypercyclic.

The following theorem shows that the condition on the previous theorem actually characterizes hypercyclicity.

Theorem 7.6. Let $T$ be an countably infinite and locally finite tree and assume that $B$ is bounded on $\mathcal{L}_{0}$. If $B$ is hypercyclic, then $T$ has no free ends.

Proof. Asumme that $T$ has a free end. Let $v^{*}$ be a vertex on the free end such that $\gamma\left(v^{*}\right)=1$ and $\gamma\left(\operatorname{par}\left(v^{*}\right)\right)=1$. Then, for each $n \in \mathbb{N}$ each of the sets $\mathrm{Chi}^{n}\left(v^{*}\right)$ and $\mathrm{Chi}^{n}\left(\operatorname{par}\left(v^{*}\right)\right)$ has a unique element.

Since $B$ is hypercyclic there exists a hypercyclic vector $f$. In fact, by the density of the hypercyclic vectors, we may assume that $\|f\|<\frac{1}{2}$. Let $\chi_{\left\{v^{*}\right\}} \in \mathcal{L}_{0}$ be the characteristic funtion of $v^{*}$. By hypercyclicity of $B$, there exists $N \in \mathbb{N}$, such that

$$
\left\|B^{N} f-\chi_{\left\{v^{*}\right\}}\right\|<\frac{1}{2}
$$

But then

$$
\begin{aligned}
\left\|B^{N} f-\chi_{\left\{v^{*}\right\}}\right\| & \geq\left|\left(B^{N} f\right)^{\prime}\left(v^{*}\right)-\chi_{\left\{v^{*}\right\}}^{\prime}\left(v^{*}\right)\right| \\
& =\left|\sum_{w \in \operatorname{Chi}^{N}\left(v^{*}\right)} f(w)-\sum_{w \in \operatorname{Chi}^{N}\left(\operatorname{par}\left(v^{*}\right)\right)} f(w)-1\right| \\
& =\left|f\left(w_{N}\right)-f\left(\operatorname{par}\left(w_{N}\right)\right)-1\right|,
\end{aligned}
$$

where $w_{N}$ is the unique element in the set $\mathrm{Chi}^{N}\left(v^{*}\right)$. Hence,

$$
\left|f\left(w_{N}\right)-f\left(\operatorname{par}\left(w_{N}\right)\right)-1\right|<\frac{1}{2}
$$

and therefore

$$
\frac{1}{2}<\left|f\left(w_{N}\right)-f\left(\operatorname{par}\left(w_{N}\right)\right)\right|
$$

But, since $\|f\|<\frac{1}{2}$, we have

$$
\left|f\left(w_{N}\right)-f\left(\operatorname{par}\left(w_{N}\right)\right)\right|<\frac{1}{2},
$$

which is a contradiction. Therefore, $T$ cannot have free ends.

We summarize the previous two theorems to obtain a full characterization of the hypercyclicity of $B$.

Theorem 7.7. Let $T$ be a rooted, countably infinite and locally finite tree and assume that $B$ is bounded on $\mathcal{L}_{0}$. Then $B$ is hypercyclic if and only if $T$ has no free ends. 


\section{REFERENCES}

[1] R. F. Allen, F. Colonna and G. R. Easley, Multiplication Operators between Lipschitz-type Spaces on a Tree Int. J. Math. Math. Sci. (2011) Art. ID 472495, 36 pp.

[2] R. F. Allen, F. Colonna and G. R. Easley, Multiplication Operators on the Iterated Logarithmic Lipschitz Spaces of a Tree, Mediterr. J. Math. 9 (2012) 575-600.

[3] R. F. Allen, F. Colonna and G. R. Easley, Multiplication Operators on the Weighted Lipschitz Space of a Tree, J. Operator Theory 69 (2013) 209-231.

[4] R. F. Allen, F. Colonna and G. R. Easley, Composition Operators on the Lipschitz Space of a Tree, Mediterr. J. Math. 11 (2014) $97-108$.

[5] F. Bayart and É. Matheron, Dynamics of Linear Operators, Cambridge University Press, Cambridge, 2009.

[6] G. D. Birkhoff, Démonstration d'un théorème élémentaire sur les fonctions entières, C. R. Acad. Sci. Paris 189 (1929) 473-475

[7] P. Cartier, Fonctions harmoniques sur un arbre, in Symposia Mathematica, Vol. IX (Convegno di Calcolo delle Probabilità, INDAM, Rome, 1971) Academic Press, London, 1972.

[8] P. Cartier, Géométrie et analyse sur les arbres, in Séminaire Bourbaki, 24ème année (1971/1972), Exp. No. 407, pp. 123-40. Lecture Notes in Math., Vol. 317, Springer, Berlin, 1973.

[9] J. M. Cohen and F. Colonna, The Bloch Space of a Homogeneous Tree, Bol. Soc. Mat. Mexicana (2), 37 (1992) $63-82$.

[10] J. M. Cohen and F. Colonna, Embeddings of Trees in the Hyperbolic Disk, Complex Variables Theory Appl. 24 (1994) 311-335.

[11] F. Colonna and G. R. Easley, Multiplication Operators on the Lipschitz Space of a Tree, Integral Equations Operator Theory 68 (2010) 391-411.

[12] F. Colonna and G. R. Easley, Multiplication Operators between the Lipschitz Space and the Space of Bounded Functions on a Tree, Mediterr. J. Math. 9 (2012) 423-438.

[13] F. Colonna and R. A. Martínez-Avendaño, Some Classes of Operators with Symbol on the Lipschitz Space of a Tree, Mediterr. J. Math. 14 (2017) 1, Art. 18, 25.

[14] F. Colonna and R. A. Martínez-Avendaño, Composition Operators on the Little Lipschitz Space of a Tree. Preprint.

[15] John B. Conway, A Course in Functional Analysis, second edition, Springer-Verlag, 1990.

[16] Richard J. Fleming and James E. Jamison, Isometries on Banach spaces: function spaces, Chapman \& Hall/CRC Monographs and Surveys in Pure and Applied Mathematics, 129, Chapman \& Hall/CRC, Boca Raton, FL, 2003.

[17] Karl G. Grosse-Erdmann and Alfred Peris-Manguillot, Linear Chaos, Springer, London, 2006.

[18] Paul R. Halmos, A Hilbert space problem book, Springer-Verlag, New York-Berlin, 1982.

[19] Z. J. Jabłoński, I. B. Jung and J. Stochel, Weighted Shifts on Directed Trees, Mem. Amer. Math. Soc. 216 (2012), no. 1017.

[20] G. R. MacLane, Sequences of Derivatives and Normal Families, J. Analyse Math. 2 (1952/53) 72-87.

[21] R. A. Martínez-Avendaño, Hypercyclicity of Shifts on Weighted $\mathbf{L}^{p}$ Spaces of Directed Trees, J. Math. Anal. Appl. 446 (2017) 823-842.

[22] S. Rolewicz, On Orbits of Elements, Studia Math. 32 (1969) 17-22.

[23] A. Shields, Weighted Shift Operators and Analytic Function Theory, in Topics in operator theory, pp. 49-128. Math. Surveys, No. 13, Amer. Math. Soc., Providence, R.I., 1974.

[24] K. Zhu, Operator Theory in Function Spaces, Second Edition, American Mathematical Society, Providence, RI, 2007.

Departamento Académico de Matemáticas, Instituto Tecnológico Autónomo de México, Mexico City, Mexico E-mail address: rubeno71@gmail.com

Centro de Investigación en Matemáticas, Guanajuato, Mexico

E-mail address: emmanuel.rivera@cimat.mx 